\title{
Aggregated Fuzzy Answer Set Programming
}

\author{
Jeroen Janssen · Steven Schockaert • Dirk \\ Vermeir - Martine De Cock
}

Received: date / Accepted: date

\begin{abstract}
Fuzzy Answer Set programming (FASP) is an extension of answer set programming (ASP), based on fuzzy logic. It allows to encode continuous optimization problems in the same concise manner as ASP allows to model combinatorial problems. As a result of its inherent continuity, rules in FASP may be satisfied or violated to certain degrees. Rather than insisting that all rules are fully satisfied, we may only require that they are satisfied partially, to the best extent possible. However, most approaches that feature partial rule satisfaction limit themselves to attaching predefined weights to rules, which is not sufficiently flexible for most real-life applications. In this paper, we develop an alternative, based on aggregator functions that specify which (combination of) rules are most important to satisfy. We extend upon previous work by allowing aggregator expressions to define partially ordered preferences, and by the use of a fixpoint semantics.
\end{abstract}

Keywords Answer Set Programming · Fuzzy Logic

\section{Introduction}

Answer Set Programming (ASP) is a declarative programming language that is especially suitable for modeling combinatorial problems. In ASP, a programmer writes rules of the form $a \leftarrow \alpha$, meaning the atom $a$ should hold whenever the conjunction of the atoms in the set $\alpha$ holds. The semantics of a set of rules, called a program, are determined by certain minimal models, named answer sets. For example, a program $P_{g c}$ solving the problem of graph coloring with two colors, viz. coloring the nodes of

J. Janssen · D. Vermeir

Dept. of Computer Science

Vrije Universiteit Brussel, VUB

Pleinlaan 2, B-1050 Brussel, Belgium

E-mail: \{jejansse,dvermeir\}@vub.ac.be

S. Schockaert · M. De Cock

Dept. of Applied Mathematics and Computer Science

Universiteit Gent, UGent

Krijgslaan 281, B-9000 Gent, Belgium

E-mail: \{steven.schockaert,martine.decock\}@ugent.be 
a graph in such a way that adjacent nodes have different colors, can be written as follows:

$$
\begin{aligned}
& \operatorname{gen}_{1}: \operatorname{white}(X) \leftarrow \operatorname{not} \operatorname{black}(X) \\
& \operatorname{gen}_{2}: \operatorname{black}(X) \leftarrow \operatorname{not} \operatorname{white}(X) \\
& \operatorname{sim}_{1}: \operatorname{sim}(X, Y) \leftarrow \text { white }(X), \text { white }(Y) \\
& \operatorname{sim}_{2}: \operatorname{sim}(X, Y) \leftarrow \operatorname{black}(X), \operatorname{black}(Y) \\
& \text { constr }: \quad \operatorname{edge}(X, Y), \operatorname{sim}(X, Y)
\end{aligned}
$$

This program is written in the generate-define-test style of ASP programs. Rules $g e n_{1}$ and gen $_{2}$ are the generate rules, which generate a certain graph coloring by stating that in every possible solution a node is either white or black. Rules $\operatorname{sim}_{1}$ and $\operatorname{sim}_{2}$ are the defining rules, which define when two nodes have a similar color. Rule constr is the test part that eliminates solutions in which two adjacent nodes have the same color. To encode a specific problem instance, rules of the form $\operatorname{fact}_{a, b}$ : edge $(a, b) \leftarrow$, denoting that there is an edge between node $a$ and $b$, are added to the above program. The resulting rules are then grounded, which means that a rule such as $g e n_{1}$ is replaced by a set of rules of the form $\left\{\operatorname{gen}_{1 a}:\right.$ white $(a) \leftarrow \operatorname{not} \operatorname{black}(a) \mid a \in$ Nodes $\}$, where Nodes is the set of nodes of the problem instance. Note that the specific substitution of the variables is denoted using a subscript. The grounded program is then handed off to an answer set solver such as Smodels [71] or DLV [46], which generates the answer sets of the program, if they exist.

For example, consider the graph depicted in Figure 1a. The answer set solver will generate two answer sets, viz. $A_{1}=\{\operatorname{edge}(a, b), \operatorname{edge}(b, a), \operatorname{sim}(a, a), \operatorname{sim}(b, b)$, white $(a)$, black $(b)\}$ and $A_{2}=\{\operatorname{edge}(a, b), \operatorname{edge}(b, a), \operatorname{sim}(a, a), \operatorname{sim}(b, b), \operatorname{black}(a)$, white $(b)\}$, that correspond to the solutions of the problem instance. Note that for overconstrained problems with no solutions, it is possible that no answer sets exist. For example, for the graph depicted in Figure 1b, no 2-coloring of the graph exists, which is reflected by the non-existence of answer sets for the corresponding program.

In recent years ASP has been extended to handle problems with imperfect information. Most notable are the probabilistic $[12,28,50,51,61,62,74]$ and possibilistic $[2,5$, $63,64]$ extensions to handle uncertainty, the fuzzy extensions $[8,33,52-56,67,74,80-82]$ which allow to encode the intensity to which the predicates are satisfied, and, more generally, many-valued extensions $[10,11,13-15,24,26,38-41,43-45,47,48,60,70,72,73$, $75,76]$.

In this paper we focus on fuzzy answer set programming (FASP). This extension allows to encode continuous optimization problems in a concise manner, similar to how ASP is able to encode discrete optimization problems. In FASP, rules take on the form $a \leftarrow f\left(b_{1}, \ldots, b_{n} ; c_{1}, \ldots, c_{m}\right)$, where $a, b_{i}$ (for $1 \leq i \leq n$ ), and $c_{j}$ (for $1 \leq j \leq m$ ) are atoms and $f$ is a $[0,1]^{n+m} \rightarrow[0,1]$ function that is monotonically increasing in its $n$ first and monotonically decreasing in its $m$ last arguments. Such a rule is satisfied when the truth value in $[0,1]$ that is attached to $f\left(b_{1}, \ldots, b_{n} ; c_{1}, \ldots, c_{m}\right)$ is lower than or equal to the truth value attached to $a$. Answer sets are certain fuzzy sets of atoms, i.e. mappings from atoms to $[0,1]$. For example, consider a continuous weighted graph coloring problem, where colors are grey values, and nodes that are adjacent need to be as different in color as possible. In FASP we can model this problem using the following program $P_{f g c}$ : 


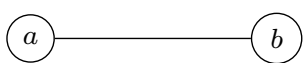

(a)

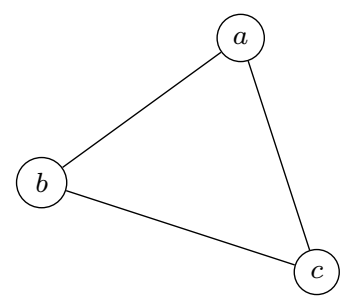

(b)

Fig. 1: Example instances for the graph coloring problem.

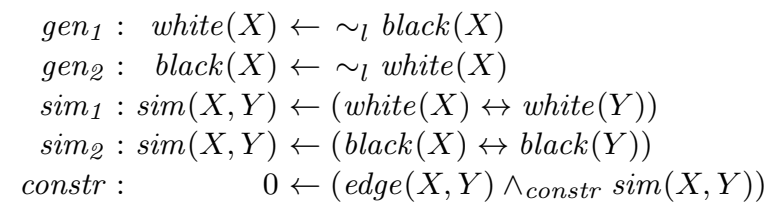

In this program we represent a grey value as a value from $[0,1]$. Hence, white $(X)$, $\operatorname{black}(X)$ and $\operatorname{sim}(X, Y)$ will be mapped to a value in $[0,1]$, but edge $(X, Y)$ is mapped to a value in $\{0,1\}$. Rules gen 1 and gen 2 are again generate rules, which generate a certain grey scale coloring. The function $\sim_{l}$ is the fuzzy negator function $\sim_{l} x=1-x$, i.e. a generalization of negation in classical logic. Rules $\operatorname{sim}_{1}$ and $\operatorname{sim}_{2}$ are the defining part of the program, which defines the similarity between the colors of adjacent nodes. The function $\leftrightarrow$ is a similarity relation defined as $x \leftrightarrow y=(x \rightarrow y) \wedge(y \rightarrow x)$, where $\wedge$ is a t-norm (a generalization of classical conjunction) and $\rightarrow$ is an implicator (a generalization of classical implication). The constr rule is a constraint which ensures edge $(X, Y) \wedge_{\text {constr }} \operatorname{sim}(X, Y) \leq 0$. The function $\wedge_{\text {constr }}$ is a t-norm. Furthermore, as edge $(X, Y)$ will be attached a value in $\{0,1\}$, this constraint ensures that any solution satisfies $\operatorname{sim}(X, Y) \leq 0$ if there is an edge between $X$ and $Y$, i.e. the colors must be as dissimilar as possible. Coloring the graph depicted in Figure 1a with $x \leftrightarrow y=$ $\min (x \rightarrow y, y \rightarrow x)$ and $\rightarrow$ an arbitrary (residual) implicator, leads to two fuzzy answer sets, viz. $M_{1}=\left\{\operatorname{edge}(a, b)^{1}, \operatorname{edge}(b, a)^{1}, \operatorname{sim}(a, a)^{1}, \operatorname{sim}(b, b)^{1}\right.$, white $\left.(a)^{1}, \operatorname{black}(b)^{1}\right\}$ and $M_{2}=\left\{\operatorname{edge}(a, b)^{1}, \operatorname{edge}(b, a)^{1}, \operatorname{sim}(a, a)^{1}, \operatorname{sim}(b, b)^{1}, \operatorname{black}(a)^{1}\right.$, white $\left.(b)^{1}\right\}$, where $M=\left\{a_{1}^{k_{1}}, \ldots, a_{n}^{k_{n}}\right\}$, with $k_{i} \in[0,1]$, means $M\left(a_{i}\right)=k_{i}$ for $i \in 1 \ldots n$, and $M(a)=0$ otherwise. Note that these correspond to the answer sets of $P_{g c}$.

Similar to the ASP program, the FASP program corresponding to the graph depicted in Figure 1b has no answer set. This is not ideal, however, as a coloring that colors node $a$ white, node $b$ black, and node $c$ grey may be better than having no solution at all. The inadmissibility is due to the constraint constr, which removes solutions where nodes have a similarity which is strictly greater than 0. A possible alternative is to allow solutions in which the similarity degree of two adjacent nodes may be greater than zero. Of course, solutions in which this degree is as small as possible are still preferred. This idea can be implemented by allowing that the last rule, constr, should not always be completely satisfied. Many of the current approaches (for example $[10,11,24,44,45,47,48,52,53,55,56,70])$ that allow partial rule satisfaction do so by coupling a weight to each rule, thus predefining to what degree each of the rules should be satisfied. 
Attaching weights to rules is not an entirely satisfactory solution, however. First of all, having weights puts an additional burden on the programmer, who, moreover, may not always be aware of which weights are suitable. Second, we are not only interested in finding any solution: if multiple solutions can be found, we are especially interested in the solution modeling the rules best. Hence, based on the degree to which the rules of the program are satisfied, it is of interest to define an ordering on the solutions, which cannot be meaningfully done using weights. In [80] the proposed solution is to attach an aggregator expression to a program. This aggregator expression maps each prospective solution to a score, based on the satisfaction degrees of the rules. In this paper, we further develop this approach. In particular, the main contribution of the paper is twofold. First, we decouple the order structure used by the aggregator expression from the lattice underlying the truth values. This ensures we can define preference orderings on answer sets which may not correspond to complete lattices. Second, our approach is based on a fixpoint semantics, rather than unfounded sets. As we show below, the approach from [80] does not correctly generalize to arbitrary truth lattices, an issue which is solved by our proposed fixpoint semantics. In addition, the fixpoint semantics are also more general, as it is not restricted to formulas that are built from t-norms. Last, the fixpoint semantics (more clearly) reveal the link between aggregated FASP and FASP approaches with weighted rules.

The structure of the paper is as follows. In Section 2 we recall the basic definitions from fuzzy set theory and fuzzy answer set programming. In section 3 we develop a fixpoint theory for fuzzy answer set programming with aggregators and investigate the main properties of this theory. While many of the properties we find are unsurprising, in the sense that they have a direct counterpart in fixpoint theory for (F)ASP, there are some notable differences as well. For instance, while one of the central properties of (F)ASP is that programs without negation have unique answer sets, it turns out that such programs can have several non-trivial answer sets in our setting, depending on the aggregator that is chosen. We apply AFASP on the reviewer assignment problem in Section 4, followed by a detailed overview of the relationship between AFASP and existing approaches in Section 5. Last, we conclude in Section 6.

A preliminary version of this paper appeared in [37].

\section{Preliminaries}

\subsection{Fuzzy Logic Connectives}

Recall that a preorder is a tuple $\mathcal{P}=(P, \leq)$ such that $(\leq) \subseteq P \times P$ is reflexive and transitive. For a specific preorder $\mathcal{P}$, we denote its ordering as $\leq_{\mathcal{P}}$. A partial order is a preorder that is anti-symmetric. A lattice $\mathcal{L}=(L, \leq)$ is a partially ordered set in which each tuple $\left(l, l^{\prime}\right) \in L^{2}$ has an infimum and supremum, denoted as $l \sqcap l^{\prime}$, respectively $l \sqcup l^{\prime}$. A lattice $\mathcal{L}=(L, \leq)$ is called bounded if $L$ has a least and greatest element, denoted as $0_{\mathcal{L}}$, respectively $1_{\mathcal{L}}$. If the lattice that is used is clear from the context we simply write 0 and 1 . A complete lattice is a lattice $\mathcal{L}=(L, \leq)$ in which each non-empty subset of $L$ has an infimum and supremum. A residuated lattice $\mathcal{L}=(L, \leq, \wedge, \rightarrow)$ is a four-tuple where $(L, \leq)$ is a bounded lattice, $\left(L, \wedge, 1_{\mathcal{L}}\right)$ is a monoid (not necessarily commutative) and the two $\mathcal{L}^{2} \rightarrow \mathcal{L}$ operators $\wedge$ and $\rightarrow$ satisfy the residuation principle, i.e. for all $x, y, z \in \mathcal{L}$ we have $x \wedge z \leq y$ iff $z \leq x \rightarrow y$. The tuple $(\wedge, \rightarrow)$ is called a residual pair. 


\begin{tabular}{|l|l|}
\hline t-norm & t-conorm \\
\hline$x \wedge_{m} y=\min (x, y)$ & $x \vee_{m} y=\max (x, y)$ \\
$x \wedge_{l} y=\max (0, x+y-1)$ & $x \vee_{l} y=\min (x+y, 1)$ \\
$x \wedge_{p} y=x \cdot y$ & $x \vee_{p} y=x+y-x \cdot y$ \\
\hline
\end{tabular}

Table 1: Common fuzzy t-norms and t-conorms over $([0,1], \leq)$

\begin{tabular}{|c|c|c|}
\hline t-norm & residual implicator & induced negator \\
\hline & if $x$ & if $x$ \\
\hline$\wedge_{m}$ & $x \rightarrow_{m} y= \begin{cases}1 & \text { otherwise }\end{cases}$ & $\sim_{m} x=\left\{\begin{array}{l}1 \\
1\end{array}\right.$ \\
\hline$\wedge_{l}$ & $x \rightarrow_{l} y=\min (1,1-x+y)$ & $\sim_{l} x=1-x$ \\
\hline$\wedge_{p}$ & $x \rightarrow_{p} y= \begin{cases}y / x & \text { if } x>y \\
1 & \text { otherwise }\end{cases}$ & $\sim_{p} x=\sim_{m} x$ \\
\hline
\end{tabular}

Table 2: Common residual pairs and induced negators over $([0,1], \leq)$

In general, fuzzy logics are logics whose semantics are defined in terms of variables that can take a truth value from some complete lattice $\mathcal{L}$ (called a truth lattice) instead of only the values true and false. Different ways exist to extend the classical logic connectives, leading to many logics with differing tautologies and axiomatizations [32, $66]$. We briefly recall the most important concepts related to fuzzy logic connectives.

A negator is a decreasing $\mathcal{L} \rightarrow \mathcal{L}$ mapping $\sim$ satisfying $\sim 0=1$ and $\sim 1=0$. A negator is called involutive iff for each $x \in \mathcal{L}$ we have $\sim(\sim x)=x$. A triangular norm (t-norm) is an increasing, commutative and associative $\mathcal{L}^{2} \rightarrow \mathcal{L}$ operator $\wedge$ satisfying for each $x \in \mathcal{L}$ the equation $1 \wedge x=x$. Intuitively, this operator corresponds to logical conjunction. A triangular co-norm (t-conorm) is an increasing, commutative and associative $\mathcal{L}^{2} \rightarrow \mathcal{L}$ operator $\vee$ satisfying for each $x \in \mathcal{L}$ the equation $0 \vee x=x$. Intuitively, this operator corresponds to logical disjunction. An implicator $\rightarrow$ is a $\mathcal{L}^{2} \rightarrow \mathcal{L}$ operator that is decreasing in its first and increasing in its second argument, satisfies $0 \rightarrow 0=1$ and for all $x \in \mathcal{L}$ satisfies $1 \rightarrow x=x$. Every t-norm $\wedge$ whose partial mappings are sup-morphisms (i.e. for which $\sup _{i}\left(x_{i} \wedge y\right)=\left(\sup _{i} x_{i}\right) \wedge y$ for any family $\left.\left(x_{i}\right)_{i \in I}\right)$ induces a residual implicator defined by $x \rightarrow y=\sup \{\lambda \in \mathcal{L} \mid x \wedge \lambda \leq y\}$ (see [16]). Furthermore, any such t-norm $\wedge$ and its residual implicator $\rightarrow$ satisfy the residuation principle. For a given implicator $\rightarrow$ its induced negator is the operator $\sim$ defined by $\sim x=x \rightarrow 0$. We summarized some common t-norms, t-conorms, residual implicators, and induced negators over the well-known complete lattice $([0,1], \leq)$ in Tables 1 and 2 .

An $\mathcal{L}$-fuzzy set $A$ in a universe $X$ is an $X \rightarrow \mathcal{L}$ mapping. When $\mathcal{L}=([0,1], \leq)$, the mapping is simply called a fuzzy set. For $x \in X$ we call $A(x)$ the membership degree of $x$ in $A$. For convenience we denote with $A=\left\{a_{1}^{k_{1}}, \ldots, a_{n}^{k_{n}}\right\}$ the fuzzy set $A$ satisfying $A\left(a_{i}\right)=k_{i}$ for $1 \leq i \leq n$ and $A(a)=0$ for $a \notin\left\{a_{1}, \ldots, a_{n}\right\}$. We use $\mathcal{F}_{\mathcal{L}}(X)$ to denote the universe of all $\mathcal{L}$-fuzzy sets in $X$. The support of a fuzzy set $A$ is defined by $\operatorname{supp}(A)=\{x \in X \mid A(x)>0\}$. Inclusion of fuzzy sets in the sense of Zadeh is defined as $A \subseteq B$ iff $\forall x \in X \cdot A(x) \leq B(x)$. 
Definition 1 Consider a complete lattice $\mathcal{L}$. A rule over $\mathcal{L}$ is an object of the form $r: a \leftarrow \alpha$ where $r$ is the label of the rule, $\leftarrow$ corresponds to a residual implicator, $a$ is either an atom or a value from $\mathcal{L}$ and $\alpha$ is of the form $f\left(b_{1}, \ldots, b_{n} ; c_{1}, \ldots, c_{m}\right)$, with $b_{i}$ and $c_{j}$ (for $1 \leq i \leq n$ and $1 \leq j \leq m$ ) either atoms or values from $\mathcal{L}$, and where $f$ is a (total and finite-time computable) $\mathcal{L}^{n+m}$ function that is increasing in its first $n$ arguments and decreasing in its $m$ last arguments. The atom $a$ is called the head of the rule and $\alpha$ is called the body. For a given rule $r$ we denote the head with $r_{h}$, the body with $r_{b}$, and the t-norm that induces the residual implicator $\leftarrow$ with $\wedge_{r}$. Furthermore we define the Herbrand Base of a rule $r$, denoted $\mathcal{B}_{r}$, as the set of atoms that occur in it. A rule with a value from $\mathcal{L}$ in its head is called a constraint, whereas a rule with a value from $\mathcal{L}$ as its body is called a fact.

When there is no cause for confusion, we will often use the label of the rule to denote the rule itself.

Definition 2 Consider a complete lattice $\mathcal{L}$. A FASP program is a finite set of rules over $\mathcal{L}$. For a given program $P$ the Herbrand Base is defined by $\mathcal{B}_{P}=\bigcup_{r \in P} \mathcal{B}_{r}$. Furthermore, we denote the lattice over which the rules in $P$ are defined with $\mathcal{L}_{P}$. An interpretation $I$ of $P$ is a $\mathcal{B}_{P} \rightarrow \mathcal{L}_{P}$ mapping. It is extended to values from $\mathcal{L}_{P}$ by $I(l)=l$ for any $l \in \mathcal{L}_{P}$, to expressions $f\left(b_{1}, \ldots, b_{n} ; c_{1}, \ldots, c_{m}\right)$ (with $b_{i}$ and $c_{j}$ in $\left.\mathcal{B}_{P} \cup \mathcal{L}_{P}\right)$ by

$$
I\left(f\left(b_{1}, \ldots, b_{n} ; c_{1}, \ldots, c_{m}\right)\right)=f\left(I\left(b_{1}\right), \ldots, I\left(b_{n}\right) ; I\left(c_{1}\right), \ldots, I\left(c_{m}\right)\right)
$$

and to rules $r: a \leftarrow \alpha \in P$ by

$$
I(r)=I(a \leftarrow \alpha)=I(a) \leftarrow I(\alpha)
$$

We say an interpretation $I$ satisfies the rule $r \in P$ to degree $k \in \mathcal{L}_{P}$ iff $I(r) \geq k$.

Note that for any residual pair $(\wedge, \rightarrow)$ it holds that

$$
I(a \leftarrow \alpha) \geq k \text { iff } I(a) \geq(k \wedge I(\alpha))
$$

due to the residuation principle. In the special case where the rule is completely satisfied, we thus obtain

$$
I(a \leftarrow \alpha)=1 \text { iff } I(a) \geq I(\alpha)
$$

Hence, residual implicators implement the intuitive requirement that, in order for a rule to be satisfied, the higher the truth degree of its body is, the higher the truth degree of its head should be.

\section{Aggregated FASP}

\subsection{Models}

Normally, given a FASP program $P$, one is interested in the interpretations $I$ satisfying for each $r \in P$ that $I(r)=1$, which are usually called models. In the present framework, however, we recognize that rules cannot always be completely fulfilled. This has 
two main advantages: first, we can tackle problems lacking a "perfect" solution (i.e. a solution satisfying all the rules to a degree of 1) and second, we can find satisfactory solutions faster if we do not need a "perfect" solution.

The first situation can occur when problems are overconstrained. For example, consider the graph coloring problem introduced in Section 1. For the graph depicted in Figure 1b, we mentioned in Section 1 that no coloring exists that will satisfy the constraint rule. Hence the problem is overconstrained and we must make some compromises, adhering stricter to rules that are considered more important. The second situation can occur when the time it takes to compute a solution is more important than finding a solution satisfying all rules.

The behavior of rules that are only partially satisfied depends crucially on the choice of the residual implicator. For example, rule $a \leftarrow \alpha$ with $k=I(a)<I(\alpha)$ is satisfied to degree $k, k \cdot I(\alpha)$ or $I(\alpha)-(1-k)$ depending on whether the Gödel resp. Goguen or Łukasiewicz implicator is used. Thus, depending on which implicator is used, the degree to which the head should be satisfied, depends 1) not at all on $I(\alpha)$; 2 ) proportionally on $I(\alpha)$; or 3 ) linearly on $I(\alpha)$. Depending on the context, each of these three situations may be required.

To create our framework, we first define rule interpretations, which are functions that map rules to a truth value (i.e. a value from the considered truth lattice).

Definition 3 Given a FASP program $P$ over the lattice $\mathcal{L}$, a rule interpretation of $P$ is a $P \rightarrow \mathcal{L}$ mapping. Any interpretation $I$ of $P$ induces a rule interpretation $\rho_{I}$ defined as $\forall(r: a \leftarrow \alpha) \in P \cdot\left(\rho_{I}(r)=I(a \leftarrow \alpha)\right)$.

Hence, the difference between the interpretation of a program and a rule interpretation is that the former maps propositional symbols to truth values, whereas the latter maps the rules themselves to a truth value. We extend the lattice ordering used to construct the rules of a program $P$ to the rule interpretations in a pointwise manner, i.e. for $\rho_{1}$ and $\rho_{2}$ rule interpretations, we define $\rho_{1} \leq \rho_{2}$ iff $\forall r \in P \cdot \rho_{1}(r) \leq \rho_{2}(r)$. The relative importance of rules is encoded in an aggregator function.

Definition 4 An aggregator over a program $P$ and preorder $\mathcal{P}$ is an order-preserving $\left(P \rightarrow \mathcal{L}_{P}\right) \rightarrow \mathcal{P}$ function.

Hence, an aggregator maps rule interpretations to preference scores from some preorder. As it is order-preserving, we guarantee that rule interpretations that map the rules to a higher degree receive a higher score. The aggregator typically encodes which rules are deemed more important by the designer, who may be more reluctant to accept solutions that do poorly on some rules while not caring much about failing to fully satisfy others.

Example 1 Consider the fuzzy graph coloring program $P_{f g c}$ and Figure $1 \mathrm{~b}$ introduced in Section 1 and suppose edge $(a, c)$ is more important than edge $(b, c)$, which is more important than edge $(a, b)$. To represent this we can use an aggregator over the preorder $\mathcal{P}=(\mathbb{R}, \leq)$ :

$$
\begin{array}{r}
\mathcal{A}(\rho)=\beta(\rho) \times\left(0.7 \cdot \rho\left(\operatorname{constr}_{(a, c)}\right)+0.7 \cdot \rho\left(\operatorname{constr}_{(c, a)}\right)+0.5 \cdot \rho\left(\operatorname{constr}_{(b, c)}\right)+\right. \\
\left.0.5 \cdot \rho\left(\operatorname{constr}_{(c, b)}\right)+0.4 \cdot \rho\left(\operatorname{constr}_{(a, b)}\right)+0.4 \cdot \rho\left(\operatorname{constr}_{(b, a)}\right)\right)
\end{array}
$$

where $\beta$ is a function mapping rule interpretations to a value in $\mathbb{R}$ that is defined as $\beta(\rho)=\left(\left(\prod\left\{\rho\left(\left(\text { gen }_{1}\right)_{a}\right), \rho\left(\left(\text { gen }_{2}\right)_{a}\right) \mid a \in\right.\right.\right.$ Nodes $\left.\}\right) \times\left(\prod\left\{\rho\left(\left(\operatorname{sim}_{1}\right)_{a, b}\right), \rho\left(\left(\operatorname{sim}_{2}\right)_{a, b}\right) \mid\right.\right.$ 
$a, b \in$ Nodes $\})) \geq 1$ ). Hence, if the generate rules or similarity rules are not satisfied to degree 1 , the score of an interpretation of the rules will always be 0 ; otherwise it is the weighted sum of the constraint rules. Now consider two rule interpretations $\rho_{1}$ and $\rho_{2}$ of this program such that $\beta\left(\rho_{1}\right)=\beta\left(\rho_{2}\right)=1$ and with the following values for the constraints:

\begin{tabular}{c|c|c|c|c|c|c} 
& constr $_{(a, b)}$ & constr $_{(b, a)}$ & constr $_{(a, c)}$ & constr $_{(c, a)}$ & constr $_{(b, c)}$ & constr $_{(c, b)}$ \\
\hline$\rho_{1}$ & 0.3 & 0.3 & 0.7 & 0.7 & 0.7 & 0.7 \\
$\rho_{2}$ & 1 & 1 & 0.5 & 0.5 & 0.5 & 0.5
\end{tabular}

Computing $\mathcal{A}\left(\rho_{1}\right)$ and $\mathcal{A}\left(\rho_{2}\right)$ we obtain $\mathcal{A}\left(\rho_{1}\right)=1.92$ and $\mathcal{A}\left(\rho_{2}\right)=2$. Hence according to this aggregator, a solution satisfying the rules to the degrees specified by rule interpretation $\rho_{2}$ is better than a solution satisfying the rules to the degrees specified by rule interpretation $\rho_{1}$.

Depending on the application, this might not be what we want. For example, the fact that edge $(a, c)$ is more important can also mean that $\rho_{1}$ should be more preferred than $\rho_{2}$, since it satisfies this rule better. This can be represented using an aggregator over the partial order $\mathcal{P}^{\prime}=\left([0,1]^{3}, \leq_{\text {lex }}\right)$ where $\left(a_{1}, a_{2}, a_{3}\right) \leq_{\text {lex }}\left(a_{1}^{\prime}, a_{2}^{\prime}, a_{3}^{\prime}\right)$ is defined as:

$$
\begin{aligned}
\left(a_{1}, a_{2}, a_{3}\right) \leq_{l e x}\left(a_{1}^{\prime}, a_{2}^{\prime}, a_{3}^{\prime}\right) \equiv & \left(\forall i \in 1 . .3 \cdot a_{i} \leq a_{i}^{\prime}\right) \\
& \vee\left(\exists i \in 1 \ldots 3 \cdot\left(a_{i}<_{\mathcal{L}} a_{i}^{\prime}\right) \wedge\left(\forall j<i \cdot a_{j}=a_{j}^{\prime}\right)\right)
\end{aligned}
$$

The aggregator is

$$
\mathcal{A}^{\prime}(\rho)= \begin{cases}(0,0,0) & \text { if } \beta(\rho)=0 \\ \left(\rho\left(\text { constr }_{(a, c)}\right), \rho\left(\text { constr }_{(b, c)}\right), \rho\left(\operatorname{constr}_{(a, b)}\right)\right) & \text { otherwise }\end{cases}
$$

Note that since $\operatorname{constr}_{(x, y)}=\operatorname{constr}_{(y, x)}$, for any $x, y \in$ Nodes, we do not need to take the constraints $\operatorname{constr}_{(b, a)}$, constr $_{(c, b)}$ and $\operatorname{constr}_{(c, a)}$ into account. Using $\mathcal{A}^{\prime}$, we obtain $\mathcal{A}^{\prime}\left(\rho_{1}\right)=(0.7,0.7,0.3)$ and $\mathcal{A}^{\prime}\left(\rho_{2}\right)=(0.5,0.5,1)$, from which we obtain that $\mathcal{A}^{\prime}\left(\rho_{2}\right) \leq_{\text {lex }} \mathcal{A}^{\prime}\left(\rho_{1}\right)$, i.e. $\rho_{1}$ will indeed be strictly preferred over $\rho_{2}$.

Many aggregation strategies have been proposed over the years (for an overview see [18]). Formally, an aggregation operator $\mathcal{A}$ is defined as a function mapping vectors over $\mathcal{L}^{n}$, with $\mathcal{L}$ a complete lattice, to a preorder $\mathcal{P}$. It can easily be seen that the aggregator defined in Definition 4 above fits this definition as rule interpretations correspond to vectors in $\mathcal{L}^{n}$, with $n$ the number of rules in a program. In the following, let $u=$ $\left(u_{1}, \ldots, u_{n}\right)$ and $v=\left(v_{1}, \ldots, v_{n}\right)$ be two vectors in $\mathcal{L}^{n}$. The main task of an aggregation operator is to define an ordering over the vectors in $\mathcal{L}^{n}$, which we call the induced ordering of an aggregation operator $\mathcal{A}$. Formally for an aggregation operator $\mathcal{A}$ it is defined as

$$
u \leq_{\mathcal{A}} v \equiv \mathcal{A}(u) \leq_{\mathcal{P}} \mathcal{A}(v)
$$

In some cases the aggregator is just the identity function, and thus $u \leq_{\mathcal{A}} v \equiv u \leq_{\mathcal{P}} v$, with $\leq_{\mathcal{P}}$ an ordering over vectors in $\mathcal{L}^{n}$. This is for example the case with the Pareto aggregator and lexicographical aggregator. Formally the former maps a vector to a preorder $\left(\mathcal{L}^{n}, \leq\right.$ par $)$ with $\leq$ par defined as

$$
u \leq_{\text {par }} v \equiv \forall i \in 1 \ldots n \cdot u_{i} \leq_{\mathcal{L}} v_{i}
$$


The latter maps a vector to a preorder $\left(\mathcal{L}^{n}, \leq_{l e x}\right)$ defined as

$$
u \leq_{\text {lex }} v \equiv\left(u \leq_{\text {par }} v\right) \vee\left(\exists i \in 1 \ldots n \cdot\left(u_{i}<_{\mathcal{L}} v_{i}\right) \wedge\left(\forall j<i \cdot u_{j}=v_{j}\right)\right)
$$

In other cases the aggregator maps vectors to a single value in $\left(\mathcal{L}, \leq_{\mathcal{L}}\right)$ or $(\mathbb{R}, \leq)$. Well-known aggregation operators of this form are the minimum, maximum, median, product and sum. Though these operators are useful, sometimes, we may consider the satisfaction of some rules more important, e.g. expressed using priority levels for each rule. To cope with such priority levels, weighted versions of the basic operators have been proposed, such as the weighted sum used in Example 1. For weighted minimum and maximum we refer the reader to [22,25,83]. A particularly interesting class of operators with weights are the Ordered Weighted Average (OWA) operators (see e.g. [84,85]), which encompass a wide range of aggregation operators over vectors in $[0,1]^{n}$, including the minimum, maximum and median. Formally, given a vector $u=\left(u_{1}, \ldots, u_{n}\right) \in[0,1]^{n}$, a collection of weights $\left(w_{1}, \ldots, w_{n}\right) \in[0,1]^{n}$ such that $\sum_{i} w_{i}=1$ and a permutation $\sigma$ of $u$ such that $u_{\sigma(1)} \leq \ldots \leq u_{\sigma(n)}$, an OWA operator is defined by

$$
O W A(u)=\sum_{j=1}^{n} w_{j} x_{\sigma(j)}
$$

By manipulating the weights of the OWA operator, particular aggregation operators are obtained, with the minimum and the maximum as extreme cases, corresponding to the weight vectors $(1,0, \ldots, 0)$ and $(0, \ldots, 0,1)$ respectively. Hence, the aggregated value will always be in between the minimum and the maximum of their arguments. Interestingly, the weights of an OWA operator are not associated to a source, but to an ordered position. This makes it ideal for applications where certain outliers should not be taken into account, such as for example in the judging of olympic sports, where the most extreme scores do not count for the final score. Furthermore OWA's can be used to model the demand that most of the rules should be fulfilled, or at least a few rules should be fulfilled.

Two other families of aggregation operators allow to model interaction between values, viz. the discrete Sugeno integral [77] and the Choquet integral [9]. The difference between these aggregators is that the Sugeno integral is more suitable for ordinal aggregation (where only the order of elements is important) while the Choquet integral is suitable for cardinal aggregation (where the distance between the numbers has a meaning) [18]. Interestingly, the Sugeno integral generalizes the weighted minimum and the weighted maximum, and the Choquet integral generalizes the weighted mean and the OWA operator. The downside of these operators is the high number of weights that need to be provided by the user. To aggregate $n$ values, in principle, the user needs to supply $2^{n}$ weights, which clearly is rather cumbersome. However, in some cases one can reduce the number of required weights, see for example $[1,31]$.

One can also use t-norms and t-conorms for aggregation. However, the aggregated value of these operators is not in between the minimum and the maximum, which is useful in certain applications. Two classes of operators that do have this feature can be constructed based on t-norms and t-conorms, however, viz. the exponential compensatory operators [79] and the convex-linear compensatory operators [49,79]. Another related class of operators are uninorms [27], which generalize t-norms and t-conorms. Contrary to the two aforementioned compensatory operators, uninorms satisfy the full reinforcement property, i.e. the tendency that when we collect a number of high scores, the aggregated value will be greater than the maximum of 
these scores, and similarly when we collect a number of low scores, the aggregated value will be lower than the minimum of these scores. In some cases this follows the human aggregation process more closely.

Last, although the min and max operators are useful because they work in a strictly ordinal manner, the ordering induced by these operators can sometimes be too coarse. For example, using the minimum, the vectors $(0.1,0.5)$ and $(0.1,0.1)$ would be equally preferred as $\min (0.1,0.5)=\min (0.1,0.1)$. However, the first vector clearly has a better score for the second value to be aggregated. To cope with this, refinements of the induced ordering have been proposed, namely the discrimin and the leximin (see e.g. [23]). Formally the discrimin aggregator maps a vector to the corresponding element in the structure $\left(\mathcal{L}^{n}, \leq_{\text {disc }}\right)$ defined as

$$
u \leq_{d i s c} v \equiv \min \left\{u_{i} \mid i \in \mathcal{D}(u, v)\right\} \leq \min \left\{v_{i} \mid i \in \mathcal{D}(u, v)\right\}
$$

where $\mathcal{D}(u, v)=\left\{i \mid i \in \mathbb{N}, u_{i} \neq v_{i}\right\}$. Intuitively, this ordering is based on the idea that the values in which the two vectors agree are of no importance when comparing them. Decisions are thus based on the least satisfied discriminating value. The idea of the leximin aggregator is to represent vectors of satisfaction levels by ranked multi-sets of satisfaction degrees. Formally it maps a vector in $\mathcal{L}^{n}$ to the corresponding element in the structure $\left(\mathcal{L}^{n}, \leq_{\text {lexi }}\right)$ defined as

$$
u \leq_{l e x i} v \equiv \exists k \leq n \cdot \forall i<k \cdot\left(u_{\sigma(i)}=v_{\sigma(i)}\right) \wedge\left(u_{\sigma(k)} \leq v_{\sigma(k)}\right)
$$

where for a given vector $u, \sigma$ is a permutation of $u$ such that $u_{\sigma(1)} \leq \ldots \leq u_{\sigma(n)}$. Hence, two vectors are indifferent if the corresponding reordered vectors are the same. The leximin ordering is a refinement of both the minimum and the discrimin [19]. Similarly two refinements of the maximum, called discrimax and leximax, can be defined.

An aggregated FASP program then consists of a FASP program and an aggregator function over this program.

Definition 5 An aggregated FASP program (short: AFASP program) $P$ is a tuple $\langle\mathcal{R}, \mathcal{A}\rangle$, where $\mathcal{R}$ is a FASP program over a lattice $\mathcal{L}$, called the rule base, and $\mathcal{A}$ is an aggregator function over $\mathcal{R}$, and an arbitrary preorder $\mathcal{P}$. Given an AFASP program $P$ we denote its rule base as $\mathcal{R}_{P}$, its aggregator as $\mathcal{A}_{P}$, the lattice over which $\mathcal{R}_{P}$ is defined as $\mathcal{L}_{P}$ and the preorder used for the aggregator as $\mathcal{P}_{P}$. AFASP programs whose rule bodies are of the form $f\left(b_{1}, \ldots, b_{n} ;\right)$, i.e. only contain increasing functions, are called positive AFASP programs. An AFASP program whose rule base contains no constraints is called constraint-free; a positive constraint-free program is called simple. The set $\mathcal{B}_{P}=\mathcal{B}_{\mathcal{R}_{P}}$ is called the Herbrand base of $P$. Furthermore, we define the set $P_{a}$, for $a \in \mathcal{B}_{P}$, as $P_{a}=\left\{r \mid r \in \mathcal{R}_{P} \wedge\left(r_{h}=a\right)\right\}$. Last, a rule interpretation of an AFASP program is a rule interpretation of $\mathcal{R}_{P}$.

In the remainder of the paper the term program always refers to an AFASP program. Furthermore, we will use the term interpretation of a program for the interpretation of the rule base of the program.

Finally, we introduce two types of approximate models: $\rho$-rule models, which link rule interpretations to interpretations of the aggregator expression, and $k$-models, which are interpretations that induce rule interpretations whose score is at least $k$. 
Definition 6 Let $P=\left\langle\mathcal{R}_{P}, \mathcal{A}_{P}\right\rangle$ be an AFASP program. A $\rho$-rule model, with $\rho$ a rule interpretation of $P$, is an interpretation $I$ of $\mathcal{R}_{P}$ such that $\rho_{I} \geq \rho$. A $k$-model, $k \in \mathcal{P}_{P}$, of $P$ is any interpretation $I$ satisfying $\mathcal{A}_{P}\left(\rho_{I}\right) \geq k$. Lastly, we define the values $\min (P)=\mathcal{A}_{P}\left(\rho_{\perp}\right)$ and $\max (P)=\mathcal{A}_{P}\left(\rho_{\top}\right)$, where $\forall r \in \mathcal{R}_{P} \cdot \rho_{\perp}(r)=0$ and $\forall r \in \mathcal{R}_{P} \cdot \rho_{\top}(r)=1$. Intuitively these correspond to the minimal, resp. maximal value the aggregator expression can attain.

Obviously, any $\rho_{1}$-rule model $M$, with $\rho_{1}$ some rule interpretation, is also a $\rho_{2}$-rule model when $\rho_{2} \leq \rho_{1}$. Similarly any $k_{1}$-model $M$ of a program $P$, with $k_{1} \in \mathcal{P}_{P}$, will also be a $k_{2}$-model when $k_{2} \leq_{\mathcal{P}_{P}} k_{1}$.

Example 2 Consider program $P_{f g c}$ and the graph depicted in Figure 1b from Section 1, together with rule interpretations $\rho_{1}$ and $\rho_{2}$ from Example 1. Furthermore for this example we define $x \leftrightarrow y=\left(x \rightarrow_{m} y\right) \wedge_{m}\left(y \rightarrow_{m} x\right), \wedge_{\text {constr }}=\wedge_{l}$ and interpret the rules using the Eukasiewicz implication. To have a $\rho_{1}$-model, we need an interpretation $I$ such that $\rho_{I} \geq \rho_{1}$. Now consider

$$
\begin{aligned}
I_{1} & =\left\{\text { edge }(a, b)^{1}, \text { edge }(b, a)^{1}, \text { edge }(a, c)^{1}, \text { edge }(c, a)^{1}, \text { edge }(b, c)^{1}, \text { edge }(c, b)^{1}, \text { white }(a)^{1},\right. \\
& \text { white }(b)^{0.7}, \operatorname{black}(b)^{0.3}, \text { white }(c)^{0.3}, \operatorname{black}(c)^{0.7}, \operatorname{sim}(a, b)^{0.7}, \operatorname{sim}(b, a)^{0.7}, \operatorname{sim}(a, c)^{0.3}, \\
& \left.\operatorname{sim}(c, a)^{0.3}, \operatorname{sim}(b, c)^{0.3}, \operatorname{sim}(c, b)^{0.3}, \operatorname{sim}(a, a)^{1}, \operatorname{sim}(b, b)^{1}, \operatorname{sim}(c, c)^{1}\right\}
\end{aligned}
$$

Clearly $I_{1}(r)=1$ for every rule not in $\left\{\operatorname{constr}_{(x, y)} \mid x, y \in\right.$ Nodes $\}$, hence $I_{1}(r) \geq \rho_{1}(r)$. For the constraint rules we obtain:

$$
I_{1}\left(\operatorname{constr}_{(a, b)}\right)=\left(1 \wedge_{l} 0.7\right) \rightarrow_{l} 0=0.3=\rho_{1}\left(\operatorname{constr}_{(a, b)}\right)
$$

Likewise for the other constraint rules we obtain that $I_{1}\left(\operatorname{constr}_{(x, y)}\right)=\rho_{1}\left(\operatorname{constr}_{(x, y)}\right)$ for any $x, y \in$ Nodes. Hence $I_{1}$ is a $\rho_{1}$-rule model of $P_{f g c}$. Analogously we can verify that $I_{1}$ is not a $\rho_{2}$-rule model of $P_{f g c}$ as $I_{1}\left(\operatorname{constr}_{(a, b)}\right)=0.3<\rho_{2}\left(\operatorname{constr}_{(b, c)}\right)$. Consider now

$$
\begin{aligned}
I_{2} & =\left\{\text { edge }(a, b)^{1}, \text { edge }(b, a)^{1}, \text { edge }(a, c)^{1}, \text { edge }(c, a)^{1}, \text { edge }(b, c)^{1}, \text { edge }(c, b)^{1}, \text { white }(a)^{1},\right. \\
& \text { black }(b)^{1}, \text { white }(c)^{0.5}, \operatorname{black}(c)^{0.5}, \operatorname{sim}(a, c)^{0.5}, \operatorname{sim}(c, a)^{0.5}, \operatorname{sim}(b, c)^{0.5}, \operatorname{sim}(c, b)^{0.5}, \\
& \left.\operatorname{sim}(a, a)^{1}, \operatorname{sim}(b, b)^{1}, \operatorname{sim}(c, c)^{1}\right\}
\end{aligned}
$$

Again one can easily verify that $I_{2}(r)=1$ for any rule not in $\left\{\operatorname{constr}_{(x, y)} \mid x, y \in\right.$ Nodes\}. For the constraint rules we obtain:

$$
I_{2}\left(\operatorname{constr}_{(a, b)}\right)=\left(1 \wedge_{l} 0 \rightarrow_{l} 0\right)=1 \geq \rho_{2}\left(\operatorname{constr}_{(a, b)}\right)
$$

Likewise for every other constraint rule $\operatorname{constr}_{(x, y)}$ with $x, y \in$ Nodes we obtain $I_{2}\left(\operatorname{constr}_{(x, y)}\right) \geq \rho_{2}\left(\operatorname{constr}_{(x, y)}\right)$. Hence $I_{2}$ is a $\rho_{2}$-rule model of $P_{f g c}$.

Now consider the aggregators $\mathcal{A}$ and $\mathcal{A}^{\prime}$ from Example 1. Using $\mathcal{A}$ we obtain that $I_{1}$ is an 1.92-model and $I_{2}$ is a 2-model of $P_{f g c}$, hence $I_{2}$ is preferred over $I_{1}$. However, with $\mathcal{A}^{\prime}$ we obtain that $I_{1}$ is an $(0.7,0.7,0.3)$-model of $P_{f g c}$, whereas $I_{2}$ is an $(0.5,0.5,1)$ model of $P_{f g c}$. This means that $I_{2} \leq_{l e x} I_{1}$, and thus with this aggregator interpretation $I_{1}$ is preferred over $I_{2}$ since it satisfies the more important rules to a better degree. 


\subsection{Answer Sets}

Rules in ASP implement the intuition of (non-deterministic) forward chaining. In practice, we are therefore interested in those models that are in accordance with this intuition. Effectively, there are two types of models we wish to exclude from our solution.

The first problem is the occurrence of atoms with a value above the one warranted by the rules. Consider the rule $r: a \leftarrow \alpha$. This rule will be fully satisfied by an interpretation $I$ (i.e. have a value of 1 ) whenever $I(a) \geq I(\alpha)$. Examples are the two 1-models $M$ and $M^{\prime}$, satisfying $M(a)=1, M(\alpha)=0.5=M^{\prime}(\alpha)$ and $M^{\prime}(a)=0.5$. However, the first model attaches a higher value to $a$ than what the rule actually supports (viz. 0.5) and is therefore unwanted. In other words, we do not want to conclude anything more than what is needed to make the rule completely satisfied.

The second problem arises when atoms are "self-motivating", i.e. their truth value is supported by some rule, but that support is ultimately based on the value of the atom itself. An illustration of this can be seen in the following two-rule program $P$ with $\mathcal{A}_{P}(\rho)=\inf \left\{\rho(r) \mid r \in \mathcal{R}_{P}\right\}$ :

$$
\begin{aligned}
& r_{1}: a \leftarrow b \\
& r_{2}: b \leftarrow a
\end{aligned}
$$

Both the models $M=\left\{a^{1}, b^{1}\right\}$ and $M^{\prime}=\left\{a^{0}, b^{0}\right\}$ are free from the first problem we mentioned, but the support given to the value of $b$ is derived from the support for the value of $a$, which is itself derived from the value of $b$. Hence, only model $M^{\prime}$ is free from knowledge not supported by the program.

The models that do not suffer from these defects will be called answer sets, and correspond to particular minimal models, as we will show later on. They are defined formally in the next sections. As the definition of answer sets for non-positive programs is an extension of the one for positive programs, we introduce them separately.

\subsubsection{Positive Programs}

To define the answer sets of positive programs we need to introduce new concepts and extend concepts from FASP to deal with the partial rule satisfaction. First we introduce the support of a rule w.r.t. some value in the lattice over which the rule ranges, which captures the idea of partial rule application. Using the support, we then extend the immediate consequence operator which allows us to derive knowledge from a program in a forward chaining manner, while still allowing for partial rule satisfaction. Then we define answer sets of positive programs and introduce some motivating propositions.

Definition 7 ( [80]) Let $r: a \leftarrow \alpha$ be a rule defined over the lattice $\mathcal{L}$ and let $I$ be an interpretation of $r$. The support of this rule w.r.t. some $c \in \mathcal{L}$ is denoted as $I_{s}(r, c)$ and is defined by

$$
I_{s}(r, c)=\inf \{y \in \mathcal{L} \mid I(\alpha) \rightarrow y \geq c\}
$$

It turns out that a characterization of this operator is easy to find ${ }^{1}$.

Proposition 1 Let $r: a \leftarrow \alpha$ be a rule defined over the lattice $\mathcal{L}$, $I$ an interpretation of $r$, and $c$ a value in $\mathcal{L}$. Then $I_{s}(r, c)=I(\alpha) \wedge_{r} c$.

\footnotetext{
1 All proofs can be found in Appendix A.
} 
Example 3 Consider the rule $r: a \leftarrow_{m} \alpha$ with interpretations $I$ and $I^{\prime}$ satisfying $I(\alpha)=$ $I^{\prime}(\alpha)=0.5, I(a)=1$ and $I^{\prime}(a)=0.5$. The support of this rule w.r.t. $\rho_{\top}(r)$ is given by $I_{s}\left(r, \rho_{\top}(r)\right)=0.5 \wedge_{m} 1=0.5$. Likewise we can compute that $I_{s}^{\prime}\left(r, \rho_{\top}(r)\right)=0.5 \wedge_{m}$ $1=0.5$. Hence, $I(a)>I_{s}\left(r, \rho_{\top}(r)\right)$ and $I^{\prime}(a)=I_{s}\left(r, \rho_{\top}(r)\right)$. This means that the interpretation of $a$ by $I^{\prime}$ is consistent with the support provided by the rule, whereas the interpretation by $I$ is strictly greater. Hence, rule $r$ cannot be used to justify the value of $a$ under interpretation $I$. Likewise we can see that $I_{s}(r, 0.9)<I(r)$, meaning $I$ attaches a value to $a$ that is higher than what is needed for satisfying this rule to a degree of 0.9 .

For simple AFASP programs, answer set semantics are determined using a "forward chaining" approach, captured in the definition of the immediate consequence operator. This operator ensures that the support of a rule is propagated to its head, which means that we derive exactly the maximal amount of knowledge contained in the program.

Definition 8 Let $\mathcal{R}$ be a set of rules over a lattice $\mathcal{L}$ with $\rho$ a corresponding rule interpretation. The immediate consequence operator $\Pi_{\mathcal{R}, \rho}$ derived from $\mathcal{R}$ and $\rho$ is a mapping from $\left(\mathcal{B}_{\mathcal{R}} \rightarrow \mathcal{L}\right)$ to $\left(\mathcal{B}_{\mathcal{R}} \rightarrow \mathcal{L}\right)$ defined for $I \in\left(\mathcal{B}_{\mathcal{R}} \rightarrow \mathcal{L}\right)$ and $a \in \mathcal{B}_{\mathcal{R}}$ as:

$$
\Pi_{\mathcal{R}, \rho}(I)(a)=\sup \left\{I_{s}(r, \rho(r)) \mid r \in \mathcal{R},\left(r_{h}=a\right)\right\}
$$

For an AFASP program $P$, we usually write $\Pi_{P, \rho}$ for $\Pi_{\mathcal{R}_{P} \backslash \mathcal{C}_{P}, \rho}$, where $\mathcal{C}_{P}$ is the set of constraint rules of $P$.

The following example illustrates the use of this operator.

Example 4 Let $P$ be an AFASP program with the following rule base $\mathcal{R}_{P}$ :

$$
\begin{aligned}
& r_{1}: a \leftarrow_{m} 0.8 \\
& r_{2}: c \leftarrow_{m} 0.5 \\
& r_{3}: b \leftarrow_{m} a \wedge_{m} c \\
& r_{4}: b \leftarrow_{m} 0.2
\end{aligned}
$$

and aggregator function $\mathcal{A}_{P}(\rho)=\inf \left\{\rho(r) \mid r \in \mathcal{R}_{P}\right\}$. Consider now the interpretation $\emptyset$, which attaches to each atom the value $0_{\mathcal{L}}$. When using the rule interpretation $\rho_{\top}$ we can compute $\Pi_{P, \rho_{\top}}(\emptyset)$ as follows (note that we are using Proposition 1 in the computation):

1. For $a: \Pi_{P, \rho_{\top}}(\emptyset)(a)=\sup \left\{\emptyset_{s}\left(r, \rho_{\top}(r)\right) \mid r \in P_{a}\right\}=\emptyset_{s}\left(r_{1}, \rho_{\top}\left(r_{1}\right)\right)=0.8 \wedge_{m} 1=0.8$

2. For $b: \Pi_{P, \rho_{\top}}(\emptyset)(b)=\sup \left\{\emptyset_{s}\left(r, \rho_{\top}(r)\right) \mid r \in P_{b}\right\}=\sup \left\{\emptyset_{s}\left(r_{3}, \rho_{\top}\left(r_{3}\right)\right), \emptyset_{s}\left(r_{4}, \rho_{\top}\left(r_{4}\right)\right)\right\}$ $=\sup \left\{\emptyset\left(\left(a \wedge_{m} c\right) \wedge_{l} 1\right),\left(0.2 \wedge_{m} 1\right)\right\}=\sup \{0,0.2\}=0.2$

3. For $c: \Pi_{P, \rho_{\top}}(\emptyset)(c)=\sup \left\{\emptyset_{s}\left(r, \rho_{\top}(r)\right) \mid r \in P_{c}\right\}=0.5 \wedge_{m} 1=0.5$

Hence $\Pi_{P, \rho \top}(\emptyset)=\left\{a^{0.8}, b^{0.2}, c^{0.5}\right\}$. For rule interpretation $\rho=\left\{r_{1}^{0.5}, r_{2}^{0.3}, r_{3}^{1}, r_{4}^{1}\right\}$ the computation of $\Pi_{P, \rho}(\emptyset)$ is as follows:

1. For $a: \Pi_{P, \rho}(\emptyset)(a)=\emptyset_{s}\left(r_{1}, \rho\left(r_{1}\right)\right)=0.8 \wedge_{m} 0.5=0.5$

2. For $b: \Pi_{P, \rho}(\emptyset)(b)=\sup \left\{\emptyset_{s}\left(r_{3}, \rho\left(r_{3}\right)\right), \emptyset_{s}\left(r_{4}, \rho\left(r_{4}\right)\right\}=\sup \left\{\emptyset\left(\left(a \wedge_{m} c\right) \wedge_{l} 1\right),\left(0.2 \wedge_{m}\right.\right.\right.$ 1) $\}=\sup \{0,0.2\}=0.2$

3. For $c: \Pi_{P, \rho}(\emptyset)(c)=\emptyset_{s}\left(r_{2}, \rho\left(r_{2}\right)\right)=0.5 \wedge_{m} 0.3=0.3$ 
Hence $\Pi_{P, \rho}(\emptyset)=\left\{a^{0.5}, b^{0.2}, c^{0.3}\right\}$.

This operator is similar to the one proposed by Damásio et al. in [10]. The difference is that we add the weights of the program as a parameter of the operator, where in [10] the weights of the program are fixed in the program itself. Once we have chosen a particular rule interpretation, however, the two operators are equivalent. Hence, as in [10], our operator is monotonic for simple programs:

Proposition 2 ( [10]) Let $P$ be a positive AFASP program and $\rho$ a rule interpretation of this program. The immediate consequence operator $\Pi_{P, \rho}$ is monotonically increasing, i.e. for every two interpretations $I_{1}$ and $I_{2}$ it holds that

$$
I_{1} \leq I_{2} \Rightarrow \Pi_{P, \rho}\left(I_{1}\right) \leq \Pi_{P, \rho}\left(I_{2}\right)
$$

The following proposition shows that our operator is also monotonic in the rule interpretations, something that is also illustrated in Example 4.

Proposition 3 Let $P$ be a positive AFASP program. The immediate consequence operator is monotonically increasing in the rule interpretations, i.e. for any two rule interpretations $\rho_{1}$ and $\rho_{2}$ and interpretation $I$ of $P$ it holds that

$$
\rho_{1} \leq \rho_{2} \Rightarrow \Pi_{P, \rho_{1}}(I) \leq \Pi_{P, \rho_{2}}(I)
$$

Due to a result from Tarski [78], which states that the fixpoints of any order preserving function over a complete lattice must form a complete lattice, it follows that our consequence operator has a least fixpoint $\Pi_{P, \rho}^{*}$ for any positive AFASP program $P$ and rule interpretation ${ }^{2} \rho$. This least fixpoint can be computed using an "iterated fixpoint procedure", i.e. by applying $\Pi_{P, \rho}$ repeatedly, starting from the minimal interpretation $\emptyset$, until a fixpoint is found.

Definition 9 Let $P$ be a positive AFASP program, and let $\rho$ be a rule interpretation of $P$. Formally, we define the sequence $\mathcal{S}\langle P, \rho\rangle=\left\langle J_{i}\right| i$ an ordinal $\rangle$ by

$$
J_{i}= \begin{cases}\emptyset & \text { if } i=0 \\ \Pi_{P, \rho}\left(J_{i-1}\right) & \text { if } i \text { is a successor ordinal } \\ \bigcup_{j<i}\left(J_{j}\right) & \text { if } i \text { is a limit ordinal }\end{cases}
$$

where $\bigcup_{i \in I}\left(J_{i}\right)=\sup _{i \in I} J_{i}$.

The least fixpoint of $\Pi_{P, \rho}$ is the first element $J_{i}$ in the sequence $\mathcal{S}\langle P, \rho\rangle$ for which $\Pi_{P, \rho}\left(J_{i}\right)=J_{i}$.

Example 5 Consider program $P$ from Example 4 . If we apply $\Pi_{P, \rho \top}$ to the interpretation $J_{1}=\Pi_{P, \rho \top}(\emptyset)=\left\{a^{0.8}, b^{0.2}, c^{0.5}\right\}$ of the sequence $\mathcal{S}\left\langle P, \rho_{\top}\right\rangle$ we obtain $J_{2}$ :

1. For $\left.a: J_{2}(a)=\Pi_{P, \rho_{\top}}\left(J_{1}\right)(a)=\sup \left\{\left(J_{1}\right)_{s}\left(r, \rho_{\top}(r)\right) \mid r \in P_{a}\right)\right\}=\left(J_{1}\right)_{s}\left(r_{1}, \rho_{\top}\left(r_{1}\right)\right)$ $=0.8 \wedge_{m} 1=0.8=J_{1}(a)$

2. For $b: J_{2}(b)=\Pi_{P, \rho \top}\left(J_{1}\right)(b)=\sup \left\{\left(J_{1}\right)_{s}\left(r, \rho_{\top}(r)\right) \mid r \in P_{b}\right\}=\sup \left\{\left(J_{1}\right)_{s}\left(r_{3}\right)\right.$, $\left.\left(J_{1}\right)_{s}\left(r_{4}\right)\right\}=\sup \left\{J_{1}\left(\left(a \wedge_{m} c\right) \wedge_{l} 1\right),\left(0.2 \wedge_{m} 1\right)\right\}=\sup \{0.5,0.2\}=0.5$

2 Note that this is only the case when the lattice is non-empty. We implicitly assume in this paper that this is always the case. 
3. For $c: J_{2}(c)=\Pi_{P, \rho_{\top}}\left(J_{1}\right)(c)=\sup \left\{\left(J_{2}\right)_{s}\left(r, \rho_{\top}(r)\right) \mid r \in P_{c}\right\}=\left(J_{1}\right)_{s}\left(r_{2}, \rho_{\top}\left(r_{2}\right)\right)=$ $0.5 \wedge_{m} 1=0.5$

Hence $J_{2}=\left\{a^{0.8}, b^{0.5}, c^{0.5}\right\}$. One can readily verify that $J_{3}=\Pi_{P, \rho \top}\left(J_{2}\right)=J_{2}$. Hence $J_{2}$ is a fixpoint of $\Pi_{P, \rho_{\top}}$ and, as it is the first fixpoint of the sequence $\mathcal{S}\left\langle P, \rho_{\top}\right\rangle$ it is the least fixpoint of $\Pi_{P, \rho \top}$. In other words $J_{2}=\Pi_{P, \rho_{\top}}^{*}$.

Example 6 Consider program $P$ from Example 4 with the rule interpretation $\rho=$ $\left\{r_{1}^{0.5}, r_{2}^{0.3}, r_{3}^{1}, r_{4}^{1}\right\}$. If we apply $\Pi_{P, \rho}$ to the interpretation $J_{1}=\Pi_{P, \rho}(\emptyset)=\left\{a^{0.5}, b^{0.2}, c^{0.3}\right\}$ of the sequence $\mathcal{S}\langle P, \rho\rangle$ we obtain $J_{2}$ :

1. For $a$ : $J_{2}(a)=\Pi_{P, \rho}\left(J_{1}\right)(a)=\sup \left\{\left(J_{1}\right)_{s}(r, \rho(r)) \mid r \in P_{a}\right\}=\left(J_{1}\right)_{s}\left(r_{1}, \rho\left(r_{1}\right)\right)=$ $0.8 \wedge_{m} 0.5=0.5$

2. For $b$ : $J_{2}(b)=\Pi_{P, \rho}\left(J_{1}\right)(b)=\sup \left\{\left(J_{1}\right)_{s}(r, \rho(r)) \mid r \in P_{b}\right\}=\sup \left\{\left(J_{1}\right)_{s}\left(r_{3}, \rho\left(r_{3}\right)\right)\right.$, $\left.\left(J_{1}\right)_{s}\left(r_{4}, \rho\left(r_{4}\right)\right)\right\}=\sup \left\{J_{1}\left(\left(a \wedge_{m} c\right) \wedge_{l} 1\right),\left(0.2 \wedge_{m} 1\right)\right\}=\sup \{0.3,0.2\}=0.3$

3. For $c: J_{2}(c)=\Pi_{P, \rho}\left(J_{1}\right)(c)=\sup \left\{\left(J_{1}\right)_{s}(r, \rho(r)) \mid r \in P_{c}\right\}=\left(J_{1}\right)_{s}\left(r_{2}, \rho\left(r_{2}\right)\right)=$ $0.5 \wedge_{m} 0.3=0.3$

Again one can readily verify that $\Pi_{P, \rho}\left(J_{2}\right)=J_{2}$ and hence $J_{2}$ is the least fixpoint of $\Pi_{P, \rho}$, i.e. $J_{2}=\Pi_{P, \rho}^{*}$. Note that $\rho \leq \rho_{\top}$ and $\Pi_{P, \rho}^{*} \leq \Pi_{P, \rho \top}^{*}$, i.e. rule interpretations that put stricter requirements on the satisfaction of rules will lead to greater fixpoints.

Note that this least fixpoint may not necessarily be found in a finite number of steps, as illustrated in the following example (due to [73]).

Example 7 Consider the program Inf, with $\mathcal{R}_{\text {Inf }}$ :

$$
r: a \leftarrow \delta(a)
$$

Where $\delta(x)=x+(1-x) / 2$. Obviously, $\delta$ is increasing and, moreover, $\forall x \in[0,1]$. $0<\delta(x) \leq 1$. The first steps of the computation of the least fixpoint of $\Pi_{\text {Inf }, \rho \top}$ are shown below:

$$
\begin{aligned}
& J_{0}=\left\{a^{0}\right\} \\
& J_{1}=\Pi_{P, \rho}\left(J_{0}\right)=\left\{a^{0.5}\right\} \\
& J_{2}=\Pi_{P, \rho}\left(J_{1}\right)=\left\{a^{0.75}\right\} \\
& J_{3}=\Pi_{P, \rho}\left(J_{2}\right)=\left\{a^{0.875}\right\} \\
& J_{4}=\Pi_{P, \rho}\left(J_{3}\right)=\left\{a^{0.9375}\right\} \\
& J_{5}=\Pi_{P, \rho}\left(J_{4}\right)=\left\{a^{0.96875}\right\} \\
& J_{6}=\Pi_{P, \rho}\left(J_{5}\right)=\left\{a^{0.984375}\right\} \\
& \ldots=\ldots
\end{aligned}
$$

Clearly, $\forall i \in \mathbb{N} \cdot J_{i}<\left\{a^{1}\right\}$, but $J_{\omega}=\bigcup_{i \in \mathbb{N}} J_{i}=\left\{a^{1}\right\}$, which is the least fixpoint $\Pi_{P, \rho \top}^{*}$.

The following proposition shows us that smaller rule interpretations yield smaller (least) fixpoints. Hence if we tighten the lower bounds imposed on the rules of a program $P$, the resulting knowledge that we can derive from $P$ using forward chaining increases. This corresponds to our intuition, as in general deriveable knowledge monotonically increases with tighter constraints, e.g. from inconsistent constraints one is able to derive anything. 
Proposition 4 Let $\rho_{1} \leq \rho_{2}$ be rule interpretations of a positive AFASP program $P$. Then $\Pi_{P, \rho_{1}}^{*} \leq \Pi_{P, \rho_{2}}^{*}$.

Consider program $P$ from Example 4 and its interpretation $J_{2}$ from Example 6 . Note that for $J_{2}$ we obtain that $J_{2}\left(r_{1}\right)=0.5 \leftarrow_{m} 0.8=0.5$, that $J_{2}\left(r_{2}\right)=0.3 \leftarrow_{m} 0.5=0.3$, that $J_{2}\left(r_{3}\right)=0.3 \leftarrow_{m}\left(0.5 \wedge_{m} 0.3\right)=1$, and that $J_{2}\left(r_{4}\right)=0.3 \leftarrow_{m} 0.2=1$, and thus, according to Definition $6, J_{2}$ is a $\rho$-rule model with $\rho$ defined as in Example 6 , i.e. $\rho=\left\{r_{1}^{0.5}, r_{2}^{0.3}, r_{3}^{1}, r_{4}^{1}\right\}$. It turns out that this is a general property, i.e. that fixpoints of the immediate consequence operator are $\rho$-rule models. Note that this property holds for all constraint-free programs and thus in particular also for non-positive programs.

Proposition 5 Let $P$ be a constraint-free AFASP program, $\rho$ a rule interpretation of $P$ and $M$ a fixpoint of $\Pi_{P, \rho}$. Then $M$ is a $\rho$-rule model of $P$.

The converse is not true in general, as one can see from the following example.

Example 8 Consider the following simple AFASP program:

$$
r: a \leftarrow 0.5
$$

with rule interpretation $\rho_{\top}$ and interpretation $I=\left\{a^{1}\right\}$. As $I(r)=1=\rho_{\top}(r), I$ is a $\rho_{\top}$-rule model of $P$. But $\Pi_{P, \rho_{\top}}(I)(a)=0.5 \wedge_{m} 1=0.5<I(a)$ and thus $I$ is not a fixpoint of $\Pi_{P, \rho_{\top}}$.

However, the converse of Proposition 5 turns out to hold for minimal (w.r.t. Zadeh inclusion) $\rho$-rule models and simple programs.

Proposition 6 Let $P$ be a simple AFASP program, $\rho$ a rule interpretation of $P$ and $M$ a minimal $\rho$-rule model of $P$. Then $M$ is a fixpoint of $\Pi_{P, \rho}$.

Finally, we define $k$-answer sets of a program $P$ as those least fixpoints of the consequence operator that are $k$-models of $P$.

Definition 10 Let $P$ be a positive AFASP program. An interpretation $M$ is a $k$ answer set $\left(k \in \mathcal{P}_{P}\right)$ of $P$ iff $M=\Pi_{P, \rho_{M}}^{*}$ and $\mathcal{A}_{P}\left(\rho_{M}\right) \geq k$.

Example 9 Consider program $P$ from Example 4 and the least fixpoints $\Pi_{P, \rho_{\top}}^{*}$ and $\Pi_{P, \rho}^{*}$ (with $\rho$ as in Example 6) computed in Example 5 and Example 6 respectively. For convenience we refer to $\Pi_{P, \rho_{\top}}^{*}$ as $A_{1}$ and to $\Pi_{P, \rho}^{*}$ as $A_{2}$. Then $A_{1}$ is a 1 -answer set of $P$ as $\mathcal{A}_{P}\left(\rho_{A_{1}}\right)=\inf \left\{r_{1}, r_{2}, r_{3}, r_{4}\right\}\left(\rho_{A_{1}}\right)=1$ and $A_{2}$ is an 0.3 -answer set as $\mathcal{A}_{P}\left(\rho_{A_{2}}\right)=\inf \left\{r_{1}, r_{2}, r_{3}, r_{4}\right\}\left(\rho_{A_{2}}\right)=0.3$. Note that $A_{1}$ is also an 0.3 -answer set, since according to Definition 6 it is an 0.3-model, i.e. $\rho_{A_{1}}\left(\mathcal{A}_{P}\right) \geq 0.3$.

The idea behind this definition is that answer sets represent the knowledge inferrable from a program $P$ without resorting to external knowledge, i.e. knowledge not contained in the program. This is reflected in the definition since the least fixpoint of $\Pi_{P, \rho_{I}}$ corresponds to the result of applying forward chaining on the minimal interpretation. Furthermore, the knowledge expressed by an answer set is also maximal as it is a fixpoint of the immediate consequence operator. Hence using forward chaining on this model will not yield new knowledge.

The $k$-prefix allows to distinguish between approximate answer sets, i.e. answer sets that do not fulfill the rules of the program completely. This allows us to handle 
conflicting information, or to find approximate solutions to problems encoded as fuzzy answer set programs, when the computation of perfect solutions is too costly.

Note that answer sets for positive programs, contrary to the classical case and non-aggregated FASP approaches, are not necessarily unique. This is illustrated in the following example.

Example 10 Consider a program $P$ with the following rules:

$$
\begin{aligned}
& r_{1}: a \leftarrow m 1 \\
& r_{2}: b \leftarrow m 1
\end{aligned}
$$

and aggregator function $\mathcal{A}_{P}(\rho)=\inf \left\{\rho(r) \mid r \in \mathcal{R}_{P}\right\}$. Consider now two interpretations of $P$, viz. $I_{1}=\left\{a^{0.5}, b^{1}\right\}$ and $I_{2}=\left\{a^{1}, b^{0.5}\right\}$. It is easy to see that both of them are least fixpoints of the immediate consequence operator with their induced rule interpretations. As $\rho_{I_{1}}\left(r_{1}\right)=0.5=\rho_{I_{2}}\left(r_{2}\right)$ and $\rho_{I_{1}}\left(r_{2}\right)=1=\rho_{I_{2}}\left(r_{1}\right)$, both of them are 0.5 -answer sets.

However, since there is only one rule interpretation satisfying all the rules to degree 1 , the optimal answer set will always be unique. There is a strong connection between minimal $\rho$-rule models and the answer sets we define here, as illustrated by the following proposition.

Proposition 7 Let $P$ be a simple AFASP program and $\rho$ a rule interpretation of $P$. Then $\Pi_{P, \rho}^{*}$ is the unique minimal $\rho$-rule model of $P$.

From this proposition we can show that our answer sets correspond to minimal rule models.

Corollary 1 Let $P$ be a simple AFASP program. Then $A$ is a $\mathcal{A}_{P}\left(\rho_{A}\right)$-answer set of $P$ iff $A$ is the unique minimal $\rho_{A}$-rule model of $P$.

One may wonder whether every rule interpretation $\rho$ for a simple AFASP program $P$ can be used to generate an answer set $M=\Pi_{P, \rho}^{*}$ such that $\rho_{M}=\rho$. The answer is negative, as one can see from the following example:

Example 11 Consider the program $P$ with aggregator $\mathcal{A}_{P}(\rho)=\inf \left\{\rho(r) \mid r \in \mathcal{R}_{P}\right\}$ and the following rule base $\mathcal{R}_{P}$ :

$$
\begin{aligned}
& r_{1}: a \leftarrow_{m} 0.2 \\
& r_{2}: a \leftarrow_{m}(a>0)
\end{aligned}
$$

where $(x>0)=0$ if $x \leq 0$ and $(x>0)=1$ otherwise. Computing $\Pi_{P, \rho}^{*}$ for $\rho=$ $\left\{r_{1}^{0.2}, r_{2}^{1}\right\}$ yields $\Pi_{P, \rho}^{*}=\left\{a^{1}\right\}=M$, which induces $\rho_{M}=\left\{r_{1}^{1}, r_{2}^{1}\right\} \neq \rho$. One can easily verify that $M=\Pi_{P, \rho_{M}}^{*}$ and $\mathcal{A}_{P}\left(\rho_{M}\right) \geq 1$, thus $M$ is an 1 -answer set of $P$.

As one can see, the least fixpoint of $\Pi_{P, \rho}$ from Example 11 turned out to be a 1answer set of $P$, where $1>\mathcal{A}_{P}(\rho)$, since $\mathcal{A}_{P}(\rho)=0.2$. The following propositions show that in general the least fixpoint of $\Pi_{P, \rho}$ for some program $P$ and an arbitrary rule interpretation $\rho$ of this program will always be a $k$-answer set, for $k \geq \mathcal{A}_{P}(\rho)$. This means that to obtain a $k$-answer set of a positive program $P$ for an arbitrary $k \in \mathcal{P}_{P}$, we only need to compute $\Pi_{P, \rho}^{*}$ for some rule interpretation $\rho$ satisfying $\mathcal{A}_{P}(\rho) \geq k$. 
Proposition 8 Let $P$ be a simple AFASP program, $\rho$ a rule interpretation of $P$, and $M=\Pi_{P, \rho}^{*}$. Then $\Pi_{P, \rho_{M}}^{*}=M$.

Proposition 9 Let $P$ be a simple AFASP program and $\rho$ a rule interpretation of $P$. Then $M=\Pi_{P, \rho}^{*}$ is a $\mathcal{A}_{P}(\rho)$-answer set.

We obtain two immediate corollaries, the first of which shows that a model of a simple program is a $k$-answer set iff it is produced by some $\rho$-rule interpretation with $\mathcal{A}_{P}(\rho) \geq$ $k$.

Corollary $2 M$ is a $k$-answer set of a simple AFASP program $P$ iff there is some rule interpretation $\rho$ for which $\mathcal{A}_{P}(\rho) \geq k$, such that $M=\Pi_{P, \rho}^{*}$.

The following corollary shows that it is easy to obtain a suitable rule interpretation for simple AFASP programs.

Corollary 3 Every simple AFASP program $P$ has a max $(P)$-answer set, with max $(P)$ as defined in Definition 6.

Hence, when constructing a $k$-answer set of a simple program $P$, with $k \in \mathcal{P}_{P}$ and $k \leq \max (P)$, we can simply use $\rho_{\top}$ and compute $\Pi_{P, \rho_{\top}}^{*}$. As any $k_{1}$-answer set of $P$ is a $k_{2}$-answer set of $P$ for $k_{1} \geq k_{2}, \Pi_{P, \rho \top}^{*}$ will be a $k$-answer set for any $k \leq \max (P)$. Hence we have arrived at a practical procedure for finding answer sets of simple programs.

Example 12 Consider an AFASP program $P$ with rule base $\mathcal{R}_{P}$ :

$$
\begin{aligned}
& r_{1}: a \leftarrow_{m} 0.8 \\
& r_{2}: b \leftarrow_{m} 0.4 \\
& r_{3}: c \leftarrow a \wedge_{m} b
\end{aligned}
$$

The aggregator is $\mathcal{A}_{P}(\rho)=\rho\left(r_{1}\right)+\rho\left(r_{2}\right)+\rho\left(r_{3}\right)$, defined over the preorder $(\mathbb{R}, \leq)$. As $\mathcal{A}_{P}\left(\rho_{\top}\right)=3$, we know that $\Pi_{P, \rho_{\top}}^{*}=\left\{a^{0.8}, b^{0.4}, c^{0.4}\right\}$ is the 3 -answer set of $P$.

\subsubsection{General programs}

Definition 10 of answer sets is only applicable to positive programs. The reason for this limitation is that the definition depends crucially on the monotonicity of the fixpoint operator. In this section, we extend the definition of answer sets to cover arbitrary programs, similar to previous FASP proposals such as [53]. One might think that the semantics of non-positive programs could again be given by minimal models, but it turns out that minimal models are unsuitable. For example, consider the following program:

$$
\begin{aligned}
& r_{1}: 0 \leftarrow \sim_{l} a \\
& r_{2}: a \leftarrow a
\end{aligned}
$$

with aggregator $\mathcal{A}_{P}(\rho)=\inf \left\{\rho(r) \mid r \in \mathcal{R}_{P}\right\}$. The minimal 1-model is $\left\{a^{1}\right\}$, but the motivation for $a$ depends on $a$ itself and thus $\left\{a^{1}\right\}$ is not acceptable. The underlying reason is that constraints should not be used as support of a atom, i.e. in (F)ASP, stating that $a$ is true is not at all the same as stating that solutions in which $a$ is false are not allowed. 
To solve this problem, we reduce the semantics of such a program $P$ to that of a simple reduct program $P^{M}$, which is called the reduct w.r.t. a candidate answer set $M$, similar to [53]. Note that this generalizes the well-known Gelfond-Lifschitz transformation from [30].

Definition 11 Let $I$ be an interpretation of an AFASP program $P$. The reduct of a rule $r: a \leftarrow f\left(b_{1}, \ldots, b_{n} ; c_{1}, \ldots, c_{m}\right)$, w.r.t. $I$, denoted as $r^{I}$, is defined by

$$
r^{I}: a \leftarrow f\left(b_{1}, \ldots, b_{n} ; I\left(c_{1}\right), \ldots, I\left(c_{m}\right)\right)
$$

Similarly, the reduct w.r.t. $I$ of an AFASP program $P$, denoted $P^{I}$, is obtained from $P$ by replacing all rules $r$ in $\mathcal{R}_{P}$ by their reduct $r^{I}$. We will also write $\mathcal{R}^{I}$, with $\mathcal{R}$ a set of rules, to denote $\left\{r^{I} \mid r \in \mathcal{R}\right\}$.

Example 13 Consider a program $P$ with rule base

$$
\begin{aligned}
& r_{1}: a \leftarrow \sim_{l} b \\
& r_{2}: b \leftarrow 0.3
\end{aligned}
$$

Then the reduct $P^{A}$ for the candidate answer set $A=\left\{a^{0.7}, b^{0.3}\right\}$ contains the following rule base:

$$
\begin{aligned}
& r_{1}^{A}: a \leftarrow 0.7 \\
& r_{2}^{A}: b \leftarrow 0.3
\end{aligned}
$$

Thus, $P^{I}$ is obtained by replacing each atom $a$ in which the body function of a rule is decreasing by its valuation $I(a)$. Obviously, $P^{I}$ is a positive AFASP program. To see that the above reduction generalizes the traditional Gelfond-Lifschitz (GL) transformation, it suffices to note that in traditional logic programming the only way to have a negative occurrence of a proposition $a$ in a rule body is via a negation-as-failure literal not $a$. The GL transformation then essentially replaces such literals with their values in the intended stable model interpretation, yielding a positive program.

Answer sets of these programs are then defined similarly as in the crisp case.

Definition 12 Let $P$ be an AFASP program. An interpretation $M$ is a $k$-answer set of $P\left(k \in \mathcal{P}_{P}\right)$ iff $M=\Pi_{P^{M}, \rho_{M}}^{*}$ and $\mathcal{A}_{P}\left(\rho_{M}\right) \geq k$.

Example 14 Consider program $P_{f g c}$ from Section 1 together with interpretations $I_{1}$ and $I_{2}$ from Example 2. It turns out that both $I_{1}$ and $I_{2}$ are approximate answer sets of this program. Indeed, for $I_{1}$ the reduct $P_{f g c}^{I_{1}}$ becomes

$$
\begin{aligned}
& \left(\text { gen }_{1}\right)_{a}: \text { white }(a) \leftarrow 1 \\
& \left(\text { gen }_{1}\right)_{b}: \text { white }(b) \leftarrow 0.7 \\
& \left(\text { gen }_{1}\right)_{c}: \text { white }(c) \leftarrow 0.3 \\
& \left(\text { gen }_{2}\right)_{a}: \operatorname{black}(a) \leftarrow 0 \\
& \left(\text { gen }_{2}\right)_{b}: \operatorname{black}(b) \leftarrow 0.3 \\
& \left(\text { gen }_{2}\right)_{c}: \operatorname{black}(c) \leftarrow 0.7 \\
& \left(\operatorname{sim}_{1}\right)_{(a, a)}: \operatorname{sim}(a, a) \leftarrow \text { white }(a) \leftrightarrow_{m} \text { white }(a) \\
& \left(\operatorname{sim}_{1}\right)_{(a, b)}: \operatorname{sim}(a, b) \leftarrow \text { white }(a) \leftrightarrow m \text { white }(b) \\
& \left(\operatorname{sim}_{1}\right)_{(a, c)}: \operatorname{sim}(a, c) \leftarrow \text { white }(a) \leftrightarrow_{m} \text { white }(c)
\end{aligned}
$$




$$
\begin{aligned}
\left(\operatorname{sim}_{1}\right)_{(b, a)}: \operatorname{sim}(b, a) & \leftarrow \text { white }(b) \leftrightarrow_{m} \text { white }(a) \\
\left(\operatorname{sim}_{1}\right)_{(b, b)}: \operatorname{sim}(b, b) & \leftarrow \text { white }(b) \leftrightarrow_{m} \text { white }(b) \\
\left(\operatorname{sim}_{1}\right)_{(b, c)}: \operatorname{sim}(b, c) & \leftarrow \text { white }(b) \leftrightarrow_{m} \text { white }(c) \\
\left(\operatorname{sim}_{1}\right)_{(c, a)}: \operatorname{sim}(c, a) & \leftarrow \text { white }(c) \leftrightarrow_{m} \text { white }(a) \\
\left(\operatorname{sim}_{1}\right)_{(c, b)}: \operatorname{sim}(c, b) & \leftarrow \text { white }(c) \leftrightarrow_{m} \text { white }(b) \\
\left(\operatorname{sim}_{1}\right)_{(c, c)}: \operatorname{sim}(c, c) & \leftarrow \operatorname{white}(c) \leftrightarrow_{m} \text { white }(c) \\
\left(\operatorname{sim}_{2}\right)_{(a, a)}: \operatorname{sim}(a, a) & \leftarrow \operatorname{black}(a) \leftrightarrow_{m} \operatorname{black}(a) \\
\left(\operatorname{sim}_{2}\right)_{(a, b)}: \operatorname{sim}(a, b) & \leftarrow \operatorname{black}(a) \leftrightarrow_{m} \operatorname{black}(b) \\
\left(\operatorname{sim}_{2}\right)_{(a, c)}: \operatorname{sim}(a, c) & \leftarrow \operatorname{black}(a) \leftrightarrow_{m} \operatorname{black}(c) \\
\left(\operatorname{sim}_{2}\right)_{(b, a)}: \operatorname{sim}(b, a) & \leftarrow \operatorname{black}(b) \leftrightarrow_{m} \operatorname{black}(a) \\
(\operatorname{sim})_{(b, b)}: \operatorname{sim}(b, b) & \leftarrow \operatorname{black}(b) \leftrightarrow_{m} \operatorname{black}(b) \\
\left(\operatorname{sim}_{2}\right)_{(b, c)}: \operatorname{sim}(b, c) & \leftarrow \operatorname{black}(b) \leftrightarrow_{m} \operatorname{black}(c) \\
\left(\operatorname{sim}_{2}\right)_{(c, a)}: \operatorname{sim}(c, a) & \leftarrow \operatorname{black}(c) \leftrightarrow_{m} \operatorname{black}(a) \\
\left(\operatorname{sim}_{2}\right)_{(c, b)}: \operatorname{sim}(c, b) & \leftarrow \operatorname{black}(c) \leftrightarrow_{m} \operatorname{black}(b) \\
\left(\operatorname{sim}_{2}\right)_{(c, c)}: \operatorname{sim}(c, c) & \leftarrow \operatorname{black}(c) \leftrightarrow_{m} \operatorname{black}(c) \\
\operatorname{constr}(a, a): 0 & \leftarrow \operatorname{edge}(a, a) \wedge_{m} \operatorname{sim}(a, a) \\
\operatorname{constr}_{(a, b)}: 0 & \leftarrow \operatorname{edge}(a, b) \wedge_{m} \operatorname{sim}(a, b) \\
\operatorname{constr}(a, c): 0 & \leftarrow \operatorname{edge}(a, c) \wedge_{m} \operatorname{sim}(a, c) \\
\operatorname{constr}(b, a): 0 & \leftarrow \operatorname{edge}(b, a) \wedge_{m} \operatorname{sim}(b, a) \\
\operatorname{constr}_{(b, b)}: 0 & \leftarrow \operatorname{edge}(b, b) \wedge_{m} \operatorname{sim}(b, b) \\
\operatorname{constr}(b, c): 0 & \leftarrow \operatorname{edge}(b, c) \wedge_{m} \operatorname{sim}(b, c) \\
\operatorname{constr}(c, a): 0 & \leftarrow \operatorname{edge}(c, a) \wedge_{m} \operatorname{sim}(c, a) \\
\operatorname{constr}(c, b): 0 & \leftarrow \operatorname{edge}(c, b) \wedge_{m} \operatorname{sim}(c, b) \\
\operatorname{constr}(c, c): 0 & \leftarrow \operatorname{edge}(c, c) \wedge_{m} \operatorname{sim}(c, c)
\end{aligned}
$$

One can easily verify that $\Pi_{P_{f g c}^{I_{1}}, \rho_{I_{1}}}^{*}=I_{1}$. Hence, combining this with the observation that $I_{1}$ is an 1.92-model of $P_{f g c}$ using aggregator $\mathcal{A}$ from Example 2, we obtain that $I_{1}$ is a 1.92 -answer set of $P$. With aggregator $\mathcal{A}^{\prime}$ from Example 2 it would be a $(0.7,0.7,0.3)$ answer set. Likewise we obtain that $I_{2}=\Pi_{P_{f g c}^{I_{2}}, \rho_{I_{2}}}^{*}$, which means $I_{2}$ is a 2 -answer set with aggregator $\mathcal{A}$ and a $(0.5,0.5,1)$-answer set with aggregator $\mathcal{A}^{\prime}$.

Intuitively, answer sets need to be self-producing, i.e. by assuming the knowledge in the answer set and starting from the empty interpretation of a program, we should only be able to infer the same set. Any such set is guaranteed to contain a maximal amount of knowledge as it is a fixpoint of the immediate consequence operator and does not contain external knowledge due to the fixpoint computation starting from the empty interpretation and assumptions that originate from this same set. Note that Definition 12 supports programs with constraints, as these only influence the $k$-score obtained in the aggregator, and not the fixpoint computation of the reduct program; 
therefore constraints can only restrict the results and cannot add atoms to solutions. A first proposition ensures that answer sets are models of a program, as we would expect.

Proposition 10 Let $M$ be a k-answer set of an AFASP program $P$. Then $M$ is a $k$-model of $P$.

A second proposition ensures that answer sets are minimal rule models of a program.

Proposition 11 Let $M$ be a k-answer set of an AFASP program $P$. Then $M$ is a minimal $\rho_{M}$-model of $P$.

As is the case for classical answer set programming, the reverse of this proposition does not hold:

Example 15 Consider the $\rho_{\top}$-rule model $M=\left\{a^{0}, b^{1}\right\}$ of the following program $P$ :

$$
r: a \leftarrow m \sim_{l} b
$$

with aggregator $\mathcal{A}_{P}(\rho)=\inf \{r \mid r \in P\}$. As any $M^{\prime} \subset M$ must satisfy $M^{\prime}(a)=0$ and $M^{\prime}(b)<1$, we obtain that $M^{\prime}(r)=M^{\prime}(a) \leftarrow\left(1-M^{\prime}(b)\right)=0 \leftarrow m\left(1-M^{\prime}(b)\right)$. Since $0 \leftarrow_{m}\left(1-M^{\prime}(b)\right) \geq 1$ only if $1-M^{\prime}(b)=0$, or $M^{\prime}(b)=1$, we obtain $M^{\prime}(r)<1$.

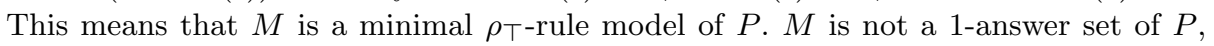
however, as $\Pi_{P^{M}, \rho \top}^{*}=\emptyset \neq M$.

It turns out that for answer sets of AFASP programs without constraints, there is a correspondence with minimal fixpoints of the non-monotonic function $\Pi_{P, \rho_{M}}$.

Proposition 12 Let $P$ be a constraint-free AFASP program with a $k$-answer set $M$. Then $M$ is a minimal fixpoint of $\Pi_{P, \rho_{M}}$.

The converse of Proposition 12 does not hold in general, however, as witnessed by the following example.

Example 16 Consider the program $P$ with rule base over $([0,1], \leq)$ :

$$
\begin{aligned}
& r_{1}: a \leftarrow a \\
& r_{2}: p \leftarrow\left(\sim_{l} p>0\right) \wedge_{m}\left(\sim_{l} a>0\right)
\end{aligned}
$$

and the following aggregator over $([0,1], \leq): \mathcal{A}_{P}=r_{1} \wedge_{m} r_{2}$. It is easy to verify that $M=\left\{a^{1}, p^{0}\right\}$, with $\rho_{M}=\rho_{\top}$, is the only, and thus minimal, fixpoint of $\Pi_{P, \rho_{\top}}$. However, $\mathcal{R}_{P^{M}}$ is

$$
\begin{aligned}
& r_{1}^{M}: a \leftarrow a \\
& r_{2}^{M}: p \leftarrow\left(1 \wedge_{m}(0>0)\right)
\end{aligned}
$$

For $P^{M}$ it thus holds that $\Pi_{P^{M}, \rho_{\top}}^{*}=\left\{a^{0}, p^{0}\right\} \neq M$. From this it follows that the minimal fixpoint of $\Pi_{P, \rho_{\top}}$ is not an answer set of $P$.

Note that the above example corresponds to our intuition about answer sets: the minimal interpretation $M$ contains an atom $a$ that is self-motivating and therefore unwanted. Hence, not every minimal fixpoint is intuitively suitable as an answer set.

Finally, we would like to point out that constraints can be simulated using nonmonotonic functions, meaning the presented results are generally applicable. We show this simulation in an upcoming paper as it is quite lengthy and does not fit the scope of this paper. 


\section{Illustrative Example}

In this section we illustrate how the features of the AFASP framework are useful for building real-life applications. The example we use is that of a "paper distribution" problem, a problem that has attracted quite some attention from the research community (see e.g. [29]). Specifically, we assume that there is a set of papers (named Papers) about a certain set of topics (named Topics) that need to be assigned to reviewers (the set of all reviewers is Reviewers) with a certain expertise on the aforementioned topics. When assigning these papers, care must be taken to ensure that there are no conflicts between reviewers and authors; furthermore, each paper should have enough reviewers and no reviewer should be burdened with a high review workload. We assume that the expertise of reviewers, the topics of papers and the affiliations of both authors and reviewers are known and thus need not be calculated with an AFASP program, but are given by a set of fact rules $\mathcal{F}$. For example to denote that reviewer $r_{1}$ is an expert of degree 0.4 on topic $t_{3}$ we add the fact expert $\left(r_{1}, t_{3}\right) \leftarrow 0.4$. The rule base $\mathcal{R}_{P_{\text {paper }}}$ of the program $P_{\text {paper }}$ solving this problem is defined over the lattice $([0,1], \leq)$ and consists of the set $\mathcal{F}$ together with the following rules:

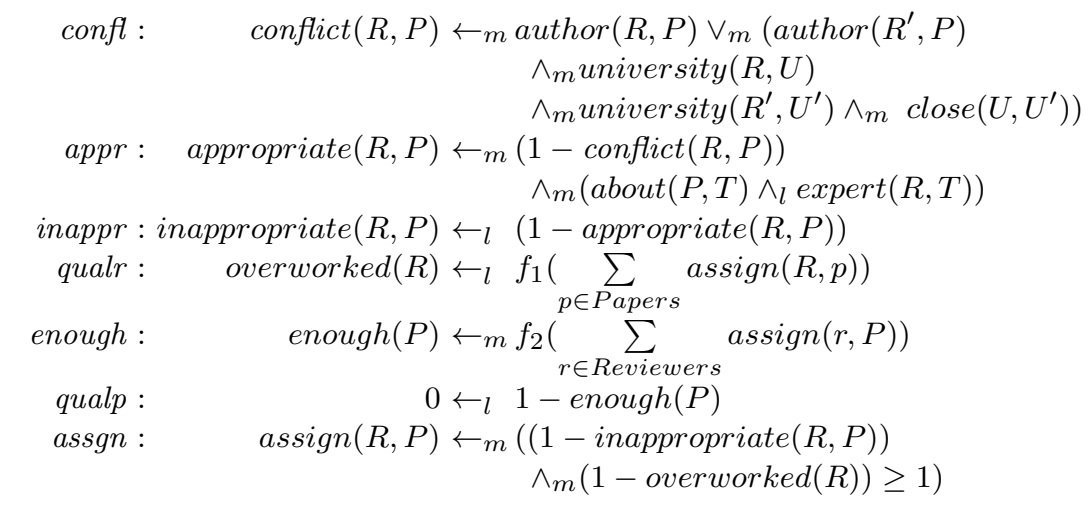

Where $f_{1}$ is:

$$
f_{1}(x)= \begin{cases}0 & \text { if } x \leq 3 \\ \frac{x-3}{7} & \text { if } x \in[4,9] \\ 1 & \text { if } x \geq 10\end{cases}
$$

and $f_{2}$ is:

$$
f_{2}(x)= \begin{cases}\frac{x}{4} & \text { if } x \leq 3 \\ 1 & \text { otherwise }\end{cases}
$$

Note that, due to the process of grounding (see e.g. [4]), a rule as inappr actually denotes the set of rules $\left\{\right.$ inappr $_{r, p}:$ inappropriate $(r, p) \leftarrow_{l}(1-\operatorname{appropriate}(r, p)) \mid$ $r \in$ Reviewers, $p \in$ Papers $\}$.

The motivation for the rules is as follows. The confl rules determine when there are potential conflicts with reviewer neutrality, i.e. conflict $(R, P)$ quantifies the degree of conflict that diminishes the suitability of person $R$ reviewing paper $P$. To keep the discussion simple, we opted to only consider the degree to which universities are close (by which we mean both geographical proximity as affiliations between universities) to 
determine these conflicts. The appr rules determine the degree to which an assignment is appropriate, based on the knowledge of the reviewer $(\operatorname{expert}(R, T))$, the topic of the paper $(\operatorname{about}(P, T))$ and potential conflicts. Note that, due to the grounding process, for a certain reviewer $r$ and paper $p$ there will be a set of rules determining the value of appropriate $(r, p)$, namely: $\left\{\operatorname{appr}_{r, p, t}:\right.$ appropriate $(r, p) \leftarrow m(1-\operatorname{conflict}(r, p))$ $\wedge_{m}\left(\operatorname{about}(p, t) \wedge_{l} \operatorname{expert}(r, t)\right) \mid t \in$ Topics $\}$. Hence, since any answer set $A$ is a fixpoint of $\Pi_{P, \rho_{A}}$ and by Definition 8 (definition of $\Pi_{P, \rho}$ for some rule interpretation $\rho$ ), this means that in any answer set $A$ it must hold that

$$
A(\operatorname{appropriate}(r, p))=\sup \left\{A_{s}\left(\operatorname{appr}, \rho_{A}(\operatorname{appr})\right) \mid \operatorname{appr} \in P_{\text {appropriate }(r, p)}\right\}
$$

We use the Lukasiewicz t-norm for combining the reviewer knowledge and paper topic to ensure that reviewers have enough knowledge about the paper content. The inappr rules determine the inappropriateness of a given paper assignment. The qualr rules determine when a reviewer is overworked, while the combination of the enough rules and qualp constraints are used to score an answer set based on the number of reviews papers have. Lastly, the assgn rules assign papers to reviewers based on the suitability of the match between reviewer and paper and bearing in mind the amount of work of reviewers.

Note that the inappr, qualr, and qualp rules are all evaluated with a Łukasiewicz implicator, while the other rules are evaluated with the Gödel implicator. The reason for this is that the former rules are allowed to be partially fulfilled and we want their fulfillment to change gradually, while the latter rules should be completely fulfilled, hence any residual implicator can be used for their evaluation. We specifically write the inappr rule to ensure that we can consider a lower inappropriateness score than the (fixed) appropriateness score when this leads to better reviewer assignments. For example, if a certain paper has a low number of reviewers, we can opt to also include a reviewer that is less familiar with the topics of the paper in this way. Furthermore, note that there is a strong interaction between the assgn, inappr, and qualr rules: if no reviewer is assigned a paper, the assgn rule is triggered for that reviewer and a paper is correspondingly assigned to him; this in turn leads to an increasing overworked score, leading to either less further assignments or a violation of the overworked constraint if this is needed to ensure that each paper has enough reviews for example.

The aggregator expression of $P$ paper then is

$$
\mathcal{A}_{P_{\text {paper }}}(\rho)=E_{1}(\rho) \cdot E_{2}(\rho) \cdot\left(E_{3}(\rho)+10 \cdot E_{4}(\rho)+20 \cdot E_{5}(\rho)\right)
$$

where

$$
\begin{aligned}
& E_{1}(\rho)=\min \{\rho(f) \mid f \in \mathcal{F}\} \geq 1 \\
& E_{2}(\rho)=\min \left\{\rho\left(\text { confl }_{r, p}\right) \wedge_{m} \rho\left(\text { appr }_{r, p}\right) \wedge_{m} \rho\left(\text { enough }_{p}\right) \wedge_{m} \rho\left(\text { assgn }_{r, p}\right)\right. \\
& \mid r \in \text { Reviewers, } p \in \text { Papers }\} \geq 1 \\
& \left.E_{3}(\rho)=\sum\left\{\rho \text { inappr }_{r, p}\right) \mid r \in \text { Reviewers }, p \in \text { Papers }\right\} \\
& E_{4}(\rho)=\sum\left\{\rho\left(\text { qualp }_{p}\right) \mid p \in \text { Papers }\right\} \\
& E_{5}(\rho)=\sum\left\{\rho\left(\text { qualr }_{r}\right) \mid r \in \text { Reviewers }\right\}
\end{aligned}
$$

The preorder for the aggregator is $(\mathbb{R}, \leq)$. The aggregation expression ensures that the confl, appr, enough, and assgn rules are completely fulfilled and allows partial 
fulfillment of the inappr, qualr, and qualp rules. The weights in the expression state that solutions in which reviewers are not overworked are better than solutions in which some reviewer is assigned a paper about a topic the reviewer is not familiar with or where papers do not have a lot of reviews. This can be seen from the fact that the qualr rules are satisfied to a lower degree when we underestimate overworked $(R)$, i.e. when we attach a lower value to overworked $(R)$ than the one warranted, when this allows us to find a solution where papers have an appropriate amount of reviewers. Thus the qualr rules are less fulfilled when reviewers are actually more overworked, meaning that giving the highest weight to these rules in the aggregator makes it more important to estimate the overworked $(R)$ values correctly.

As an example, suppose Papers $=\left\{p_{1}, \ldots, p_{10}\right\}$, Authors $=\left\{a_{1}, \ldots, a_{10}\right\}$ such that author $\left(a_{i}, p_{i}\right)=1$ for any $i \in 1 \ldots 10$ and Universities $=\left\{u_{1}, \ldots, u_{10}\right\}$ such that university $\left(a_{i}, u_{i}\right)=1$ for any $i \in 1 \ldots 10$. Furthermore, suppose Reviewers $=\left\{r_{1}, \ldots\right.$, $\left.r_{5}\right\}$, Universities ${ }^{\prime}=\left\{u_{1}^{\prime}, \ldots, u_{5}^{\prime}\right\}$ such that university $\left(r_{i}, u_{i}^{\prime}\right)=1$ for any $i \in 1 \ldots 5$. Tables 3, 4, and 5 (to be found at the end of the paper) then respectively give the about, expert and close scores. Note that for any answer set of this program, the value of these atoms must be as given in the aforementioned tables as these are added to the program as fact rules that must be completely satisfied, i.e. things that must be true in any answer set. In Tables 6 and 7 the corresponding conflict and appropriateness scores are shown. Note that, as the confl and appr scores directly depend on the atoms given as facts and since the rules defining these atoms always need to be completely satisfied, these atoms must also have the same score in any answer set of $P_{\text {paper }}$. The difference between answer sets will be in the overworked, inappropriate, enough and assign scores.

Now, consider an approximate answer set $A_{1}$ with assignments as given in Table 8 . The corresponding inappropriate scores can be found in Table 9. One can check that $A_{1}$ indeed is an answer set by checking whether $\Pi_{P^{A_{1}, \rho_{A_{1}}}}^{*}=A_{1}$. For example, for inappropriate $\left(r_{1}, p_{1}\right)$ we can see that there is only one rule with inappropriate $\left(r_{1}, p_{1}\right)$ in its head, viz. inappr $_{r_{1}, p_{1}}$. Now the reduct of this rule is

$$
\operatorname{inappr}_{r_{1}, p_{1}}^{A_{1}}: \text { inappropriate }\left(r_{1}, p_{1}\right) \leftarrow_{l} A_{1}\left(1-\operatorname{appropriate}\left(r_{1}, p_{1}\right)\right)
$$

which, by Table 7 , is equivalent to

$$
\text { inappr } r_{r_{1}, p_{1}}^{A_{1}}: \text { inappropriate }\left(r_{1}, p_{1}\right) \leftarrow_{l} 0.5
$$

We can also compute $\rho_{A_{1}}\left(\right.$ inappr $\left._{r_{1}, p_{1}}\right)$ as

$$
\rho_{A_{1}}\left(\text { inappr }_{r_{1}, p_{1}}\right)=A_{1}\left(\text { inappropriate }\left(r_{1}, p_{1}\right) \leftarrow_{l} 1-\operatorname{appropriate}\left(r_{1}, p_{1}\right)\right)
$$

which, by Table 7 and Table 9, is equal to

$$
\rho_{A_{1}}\left(\text { inappr }_{r_{1}, p_{1}}\right)=0 \leftarrow_{l} 0.5=0.5
$$

Now using (10) and (11) we know that

$$
\begin{aligned}
\Pi_{P^{A_{1}, \rho_{A_{1}}}}^{*}\left(\text { inappropriate }\left(r_{1}, p_{1}\right)\right) & =A_{1}(0.5) \wedge_{l} \rho_{A_{1}}\left(\text { inappr }_{r_{1}, p_{1}}\right) \\
& =0.5 \wedge_{l} 0.5 \\
& =0 \\
& =A_{1}\left(\text { inappropriate }\left(r_{1}, p_{1}\right)\right)
\end{aligned}
$$


One can check that for all other atoms $l \in \mathcal{B}_{P_{\text {paper }}}$ we also obtain that $\Pi_{P A_{1}, \rho_{A_{1}}}^{*}(l)=$ $A_{1}(l)$ and thus that $A_{1}$ is an answer set of $P_{\text {paper. }}$.

Now, from the inappropriate scores in Table 9, we for example already know that $\rho_{A_{1}}\left(\right.$ inappr $\left._{r_{1}, p_{1}}\right)=0.5$ by (11). Likewise we can see from Table 7 and Table 9 that

$$
\begin{aligned}
A_{1}\left(\text { inappr }_{r_{1}, p_{4}}\right) & =A_{1}\left(\text { inappropriate }\left(r_{1}, p_{4}\right) \leftarrow_{l}\left(1-A_{1}\left(\text { appropriate }\left(r_{1}, p_{4}\right)\right)\right)\right. \\
& =0.8 \leftarrow_{l} 0.8=1
\end{aligned}
$$

The values of inappr $_{r, p}$ for any $r \in$ Reviewers and $p \in$ Papers follow in the same fashion and thus we can compute

$$
E_{3}\left(\rho_{A_{1}}\right)=\sum\left\{A_{1}\left(\text { inappr }_{r, p}\right) \mid r \in \text { Reviewer }, p \in \text { Papers }\right\}=385 / 10
$$

As the enough rules should always be completely satisfied, we have that enough $(p)$ for a certain paper $p$ always should be equal to $f_{2}\left(\sum\{\operatorname{assign}(r, p) \mid r \in\right.$ Reviewer $\left.s\}\right)$. Due to each paper having three reviewers in $A_{1}$ we then know that $A_{1}(\operatorname{enough}(p))$ for any paper $p$ will be equal to $f_{2}(3)=3 / 4$. This means that for any qualp $p$ rule we have that $A_{1}\left(\right.$ qualp $\left._{p}\right)=3 / 4$. As there are 10 papers in total we can thus compute:

$$
\begin{aligned}
E_{4}\left(\rho_{A_{1}}\right) & =\sum\left\{A_{1}\left(\text { qual }_{p}\right) \mid p \in \text { Paper }\right\} \\
& =\sum\{(3 / 4) \mid p \in \text { Papers }\} \\
& =10 \cdot(3 / 4) \\
& =15 / 2
\end{aligned}
$$

Now, in this answer set we have that, even though reviewer $r_{1}$ has 6 reviews, $A_{1}$ ( overworked $(r))=0$ for each assigned reviewer $r$; thus for some reviewers we underestimate their degree of being overworked to ensure that papers have enough reviews. From the foregoing, we can compute

$$
\begin{aligned}
E_{5}\left(\rho_{A_{1}}\right) & =\sum\left\{A_{1}\left(\text { qual }_{r}\right) \mid r \in \text { Reviewer }\right\} \\
& =\sum\left\{\left(f_{1}\left(\sum\left\{A_{1}(\text { assign }(r, p)) \mid p \in \text { Paper } s\right)\right) \rightarrow_{l} 0 \mid r \in \text { Reviewers }\right\}\right. \\
& =\sum\left\{1-\left(f_{1}\left(\sum\left\{A_{1}(\text { assign }(r, p)) \mid p \in \text { Paper }\right\}\right)\right) \mid r \in \text { Reviewers }\right\} \\
& =19 / 7
\end{aligned}
$$

Hence we obtain that

$$
\begin{aligned}
\mathcal{A}_{P_{\text {paper }}}\left(\rho_{A_{1}}\right) & =E_{1}\left(\rho_{A_{1}}\right) \cdot E_{2}\left(\rho_{A_{1}}\right) \cdot\left(E_{3}\left(\rho_{A_{1}}\right)+10 \cdot E_{4}\left(\rho_{A_{1}}\right)+20 \cdot E_{5}\left(\rho_{A_{1}}\right)\right) \\
& =2349 / 14 \\
& \approx 167.8
\end{aligned}
$$

There is room for improvement, however, as this answer set clearly contains a high work burden for some reviewers, while creating a minimal workload for others. Due to the nature of our aggregator expression, we can spread the papers among reviewers, potentially giving papers to reviewers with a lower knowledge of the domain, to obtain a better answer set. Answer set $A_{2}$, for which the assignments are given in Table 8 , relieves the burden of reviewers $r_{3}$ and $r_{5}$ by assigning more reviews to reviewer $r_{4}$. Computing the value of the aggregator expression, we first obtain 
from the inappropriate scores in Table 10 that $E_{3}\left(\rho_{A_{2}}\right)=\sum\left\{A_{2}\left(\right.\right.$ inappr $\left._{r, p}\right) \mid r \in$ Reviewers, $p \in$ Papers $\}=371 / 10$; furthermore we can compute in a similar fashion as for $A_{1}$ that $E_{4}\left(\rho_{A_{2}}\right)=\sum\left\{A_{2}\left(\right.\right.$ qualp $\left._{p}\right) \mid p \in$ Papers $\}=15 / 2$ as every paper again has three reviewers assigned. Now, by calculating the assignments per reviewer we obtain $E_{5}\left(\rho_{A_{2}}\right)=\sum\left\{A_{2}\right.$ (qual $\left._{r}\right) \mid r \in$ Reviewers $\}=20 / 7$. The foregoing shows $\mathcal{A}_{P_{\text {paper }}}\left(\rho_{A_{2}}\right)=11847 / 70 \approx 169.2$, hence answer set $A_{2}$ is more suitable than $A_{1}$, as we expected.

The reader might wonder how the connectives and the input degrees in the reviewer assignment program can be determined. For the connectives an interesting approach is to first write a program that captures the overall solution and then use training data and e.g. a hill-climbing algorithm to fine-tune this program. This is similar to the techniques that are applied in rule-based systems for fuzzy control. To determine the input degrees automatic methods can be used. For example, in the field of information retrieval there are approaches that determine the expert degree of a person by means of language models (see e.g. [3]). The inputs of the reviewer assigment program can thus be seen as the outputs of such analyses.

\section{Relationship to Existing Approaches}

The combination of answer set programming and logic programming with uncertainty theories has received a great deal of attention over the past years. Among others, there have been extensions of logic programming using probabilistic reasoning $[12,28,50,51$, $61,62,74]$, possibilistic reasoning [2,63, 64], fuzzy reasoning [8,33, 52-56,67, 74, 80-82], and more general many-valued or uncertainty reasoning $[10,11,13-15,24,26,38-41$, 43-45, 47, 48, 60, 70, 72, 73, 75, 76]. Roughly, one can divide [73] these approaches in two classes, viz. implication-based (IB) and annotation-based (AB) frameworks. In the implication-based setting a rule is generally of the form

$$
a \stackrel{w}{\leftarrow} \alpha
$$

where $a$ is an atom, $\alpha$ is a body expression, and $w \in \mathcal{L}$, with $\mathcal{L}$ the lattice used for truth values. Intuitively, such a rule denotes that in any model of the program the truth degree of the implication $\alpha \rightarrow a$ must be greater than or equal to the weight $w$. In the annotation-based approaches one considers annotations, which are either constants from the truth lattice $\mathcal{L}$, variables ranging over this truth lattice, or functions over elements of this truth lattice applied to annotations. A rule is then of the form

$$
a: \mu \leftarrow b_{1}: \mu_{1}, \ldots, b_{n}: \mu_{n}
$$

where $a, a_{1}, \ldots, a_{n}$ are atoms and $\mu, \mu_{1}, \ldots, \mu_{n}$ are annotations. Intuitively, an annotated rule denotes that if the certainty of each $b_{i}, 1 \leq i \leq n$, is at least $\mu_{i}$, then the certainty of $a$ is at least $\mu$. The links between these two approaches are well-studied in e.g. $[15,39,44,45]$ and we will therefore not repeat these results. In this section, we give an overview of these related approaches and study the links between our framework and related proposals. 
5.1 Fuzzy and Many-Valued Logic Programming Without Partial Rule Satisfaction

Many proposals for fuzzy and many-valued logic programming with rules that have to be completely fulfilled have been published. In this category one finds most annotationbased approaches, e.g. $[8,38,39,67,73,76]$ and some implication-based approaches where the weight of each rule is 1 , e.g. [13-15, 41]. Some of these proposals only contain monotonic functions in rules (e.g. the AB approach from $[8,39]$ and the IB approaches from $[14,15,41]$ ), while others feature negation (e.g. the $\mathrm{AB}$ approaches from $[38,67$, $73,76]$ ) or even arbitrary decreasing functions (e.g. the IB approach from $[13,72]$ ). These proposals differ from ours as they do not incorporate the idea of partial rule satisfaction.

We can readily embed the IB approaches in our framework by supplying these programs with the infimum aggregator. When modelled like this, the 1-answer sets of the embedding will correspond exactly to the answer sets of programs from the aforementioned frameworks. Thanks to this embedding we also inherit the modelling power that is already present in some of these proposals. For example, from the embeddings shown in $[12,15]$, and using the fact that we can embed [13] in our approach, we inherit the capacity to model Generalized Annotated Logic Programs [39], Probabilistic Deductive Databases [42], Possibilistic Logic Programming [21], Hybrid Probabilistic Logic Programs $[17]^{3}$ and Fuzzy Logic Programming [81].

The AB approach from [8] is interesting in that annotations are actually fuzzy sets, which allows for an intuitive modelling language. Whether the semantics of this specific framework can be truthfully embedded in our approach is not immediately clear, but as the family of all fuzzy sets in a given universe forms a complete lattice, when equipped with Zadeh intersection and union, the use of fuzzy sets, together with functions over fuzzy sets is certainly possible in the AFASP language.

\subsection{Weighted Rule Satisfaction Approaches}

Some IB approaches to fuzzy and many-valued logic programming feature partial rule fulfillment by adding "weights" to rules (e.g. [10,11, 24, 44, 45, 47, 48, 52, 53, 55-58,70]). These weights are specified manually and they reflect the minimum degree of fulfillment required for a rule. Formally, such a rule takes on the form of

$$
a \stackrel{w}{\leftarrow} \alpha
$$

where $a$ is an atom, $\alpha$ is a body expression, $\leftarrow$ is a residual implicator over $[0,1]$ and $w$ is a value of $[0,1]$. We will use $r_{h}$ and $r_{b}$ to refer to the head, resp. the body of a rule $r$ as usual, and $r_{w}$ to refer to the weight $w$. In the case of $[24,52,53,55,56,70]$, the bodies of rules are restricted to combinations of triangular norms, possibly with negation-as-failure literals in $[52,53,55-58]$, whereas in $[10,11,44,45,47,48]$ the bodies consist of monotonically increasing functions, where some approaches do not feature non-monotonic negation $[10,11,44,45]$ and others feature negation under the wellfounded semantics [47,48]. Furthermore, [10] allows a combination of multiple lattices to be used for rules. This last feature is obtained in the AFASP setting by using the

\footnotetext{
3 Note that the translation process in [12] is exponential in the size of the program, but, as the authors point out, this is to be expected as reasoning in these programs in most cases is exponential.
} 
cartesian product of all these lattices as the lattice for program rules and using the corresponding projections to extend the operators used to this product lattice.

The semantics of a program consisting of weighted rules without negation-as-failure is defined in two ways in the literature. We will take [53] and [10] as examples of these two methods, but the following discussion equally applies to all the approaches mentioned earlier, barring some minor syntactical issues. In the case of [53], an interpretation $M$ is called a model of a program $P$ when for all $r$ in $P$ it holds that $M\left(r_{h}\right) \geq M\left(r_{b} \wedge_{r} r_{w}\right)$. Answer sets of these programs are then defined as minimal models. In [10], answer sets are defined as the least fixpoints of an immediate consequence operator, defined for a program $P$, interpretation $I$ of $P$ and atom $l \in \mathcal{B}_{P}$, as:

$$
\Pi_{P}(I)(l)=\sup \left\{I\left(r_{b} \wedge_{r} r_{w}\right) \mid r \in P_{l}\right\}
$$

It is known that these two semantics coincide, which can also be shown using the results on AFASP, as demonstrated below.

Note that due to Proposition 1 , it holds that $\Pi_{P}=\Pi_{P, \rho_{w}}$, where $\rho_{w}(r)=r_{w}$. Hence, for simple AFASP programs, the semantics of [10] can be obtained by taking the least fixpoint w.r.t. the rule interpretation corresponding to the rule weights. Furthermore, from Proposition 7 , we know that the least fixpoint of $\Pi_{P, \rho_{w}}$ corresponds to the minimal $\rho_{w}$-rule model of $P$. An interpretation $M$ is a $\rho_{w}$-rule model of $P$ iff for each rule $r \in P$ it holds that $M\left(r_{h} \leftarrow r_{b}\right) \geq \rho_{w}(r)$ and hence, by the residuation principle, that $M\left(r_{h}\right) \geq M\left(r_{b}\right) \wedge_{r} \rho_{w}(r)$. This means that $\rho_{w}$-rule models correspond to models in the sense of [53]. Hence the semantics of [53] and [10] coincide and can both be generated from a simple AFASP program.

Furthermore, due to the equivalence between $\Pi_{P}$ and $\Pi_{P, \rho_{w}}$, we can use the termination conditions from [10] to determine structural conditions that ensure that the computation of the least fixpoint of $\Pi_{P, \rho}$ ends.

Note that, different to AFASP, programs with weight rules without negation-asfailure only have a single answer set corresponding to the minimal model satisfying the rules to the stated weights. This means that the weight of a rule should not simply be seen as the minimal degree of satisfaction, but that the semantics of these programs correspond to a cautious use of the rules and their weights.

At first, one might think that these semantics can easily be embedded in the AFASP framework by moving the weights into the aggregator expression and using them as lower bounds on the satisfaction of their corresponding rules. Formally, this means that we define for a program $P$ in the sense of $[10,53]$ and rule base $\left\{r_{1}, \ldots, r_{n}\right\}$ with corresponding weights $w_{1}, \ldots, w_{n}$, the program $P^{\prime}$ with rule base $\mathcal{R}_{P^{\prime}}=\left\{r_{i}\right.$ : $\left.r_{h} \leftarrow r_{b} \mid i \in 1 \ldots n\right\}$ and aggregator expression $\mathcal{A}_{P^{\prime}}(\rho)=\left(\rho\left(r_{1}\right) \geq w_{1}\right) \wedge \ldots \wedge$ $\left(\rho\left(r_{n}\right) \geq w_{n}\right)$. The semantics of programs $P$ and $P^{\prime}$ do not coincide, however, as shown in the following example.

Example 17 Let $P$ be a program in the sense of $[10,53]$ with rule base $\mathcal{R}_{P}=\{r$ : $a \stackrel{0.5}{\leftarrow} 1\}$. It is easy to see that the unique answer set of this program is $\left\{a^{.5}\right\}$. The corresponding AFASP program $P^{\prime}$ with rule base $\mathcal{R}_{P^{\prime}}=\left\{r: a \leftarrow_{m} 1\right\}$ and aggregator expression $\mathcal{A}_{P^{\prime}}(\rho)=\rho(r) \geq 0.5$, however, has multiple $k$-answer sets for varying $k \in[0,1]$, such as the 1 -answer set $\left\{a^{1}\right\}$. The original answer set of $P$ is only an 0.5 -answer set of $P^{\prime}$.

A proper embedding of these semantics in the AFASP framework can be obtained as follows. Suppose $P$ is a program with weighted rules, then the corresponding AFASP 
program $P_{\text {weight }}$ is defined as

$$
P_{\text {weight }}=\left\{r_{h} \leftarrow r_{b} \wedge_{r} r_{w} \mid r \in P\right\}
$$

The lattice to be used for evaluating the rules is then $([0,1], \leq)$, the aggregator lattice is $(\mathbb{B}, \leq)$. The aggregator expression is given as

$$
\mathcal{A}_{P_{\text {weight }}}(\rho)=\forall r \in P_{\text {weight }} \cdot(\rho(r)=1)
$$

Example 18 Consider program $P$ from Example 17. Using the proper embedding discussed above we obtain an AFASP program $P_{\text {weight }}$ with rule base $\mathcal{R}_{P_{\text {weight }}}=\{r$ : $\left.a \leftarrow m\left(1 \wedge_{m} 0.5\right)\right\}$ and aggregator expression $\mathcal{A}_{P_{\text {weight }}}=(\rho(r)=1)$. The 1-answer set of $P_{\text {weight }}$ is now $\left\{a^{0.5}\right\}$, which corresponds to the answer set of $P$.

The following proposition shows that for simple AFASP programs, this is indeed a truthful embedding of these semantics:

Proposition 13 Let $P$ be a simple AFASP program in the sense of [10]. Then $A$ is a 1-answer set of $P_{\text {weight }}$ iff $A=\Pi_{P}^{*}$.

For approaches featuring negation-as-failure under the answer set semantics in the body of rules, we will again take [53] as a representative example. Although the approaches in $[55,56]$ are slightly different in that the reduct operation moves the value of the negated literals in the weight of the rule instead of directly substituting it in the rule body, the net result is the same. Negation-as-failure in this approach is denoted as not $\sim l$, where $l$ is an atom and $\sim$ a negator over $[0,1]$. If $P$ is a program with negationas-failure in the sense of [53], then an interpretation $A$ is called an answer set of $P$ if $A$ is the answer set of $P^{A}$, where $P^{A}$ is a generalized Gelfond-Lifschitz transformation replacing all negation-as-failure literals not $\sim l$ by the value $A(\sim l)$. It is easy to see that this Gelfond-Lifschitz transformation is a special case of the reduct (Definition 11) we introduced for AFASP programs. From this, it easily follows that the embedding mentioned before still works in the presence of negation-as-failure. Hence we obtain the following proposition.

Proposition 14 Let $P$ be a program in the sense of [53]. Then $A$ is a 1-answer set of $P_{\text {weight }}$ iff $A$ is an answer set of $P$ in the sense of [53].

Although these proposals feature partial rule satisfaction and are therefore better equipped to model real-life phenomena than previous proposals, the use of weights introduces the new problem of "weight-guessing". The AFASP framework eliminates the weight-guessing problem by using an aggregator expression, which encodes which combinations of partially satisfied rules are more desirable than others. In this light, one could think of AFASP programs as programs with variables as rule-weights instead of fixed weights, where the variables must be chosen according to the aggregator expression. In effect, due to Corollary 2 and the fact that rule interpretations are analogous to weights on rules, this means that semantically, a single AFASP program corresponds to a set of programs with weights. This shows that the aggregator expression has a substantial modeling advantage over attaching arbitrary weights.

Interestingly, in $[57,58]$, a measure is introduced that shows how the weights of the rules of a program without stable models should be increased or decreased to obtain a new program with stable models. This is related our approach, where rule weights 
are variable and can be changed to obtain approximate answer sets, if none exists. Our approach differs in the fact that we do not have predefined weights on rules and assume that the best solution is the solution satisfying all the rules best. Furthermore, due to the aggregator, we can compare approximate answer sets in our framework with a preference order that is chosen by the programmer.

\subsection{Fuzzy Answer Set Programming}

In [80], Fuzzy Answer Set Programming is introduced. Our presented approach generalizes this framework by allowing a much richer vocabulary of expressions to use in rules and by allowing more sophisticated aggregator expressions. Specifically, we allow arbitrary monotonic functions in rule bodies, whereas [80] only allows t-norms in bodies. Furthermore, we base our semantics on fixpoints, which more clearly shows the link between other FASP approaches, and also fixes a problem with the semantics of [80] when generalizing to arbitrary lattices as truth values, as demonstrated below. Moreover, it is not clear how the semantics of [80] can be extended to deal with arbitrary monotonic functions in rule bodies, where this is straightforward in our fixpoint approach.

Semantically, in [80], an answer set $M$ has a degree $k$, which, as in the present approach, reflects the value of an aggregator function that combines the degree of satisfaction of the rules in the program. However, as opposed to the present approach, this aggregator must have a value in the same lattice as the one used for the rules. As shown throughout the examples in this paper, our more general aggregator can be advantageous for modelling certain real-life problems. Furthermore, an answer set is defined in [80] as a model that is free from unfounded sets. Intuitively, the concept of unfounded set provides a direct formalization of "badly motivated" (as described in Section 3.2) conclusions.

Formally, a set $X$ of atoms is called unfounded w.r.t. an interpretation $I$ of a program $P$ iff for all $a \in X$, every rule $r: a \leftarrow \alpha \in P$ satisfies either

(i) $X \cap \alpha \neq \emptyset$, or

(ii) $I_{s}\left(a, \rho_{I}(r)\right)<I(a)$, or

(iii) $I\left(r_{b}\right)=0$

Intuitively, condition (i) above describes a circular motivation while (ii) asserts that $a$ is overvalued w.r.t. $r$. Condition (iii) is needed to ensure that the semantics are a proper generalization of the classical semantics. Answer sets to a degree $k$ are then defined in [80] as $k$-models that are free from unfounded sets, i.e. a $k$-model $M$ is a $k$-answer set iff $\operatorname{supp}(M) \cap X=\emptyset$ for any unfounded set $X$. A nice feature is that this single definition covers any program $P$, regardless of whether it is positive, or has constraint rules. One may wonder whether the concept of unfounded set can be generalized to AFASP programs. For example, a natural generalization would simply replace the circularity definition $X \cap \alpha \neq \emptyset$ above by "some element of $X$ occurs as an argument in which the body expression $\alpha$ is increasing". However, this approach fails, as illustrated by the program $P$ from Example 11 where it can easily be verified that $\{a\}$ would be unfounded w.r.t. the interpretation $\left\{a^{1}\right\}$ since $r_{1}: a \leftarrow_{m} 0.2$ satisfies (ii) above while $r_{2}: a \leftarrow_{m}(a>0)$ satisfies (i). Note that this failure is only due to the presence of $(a>0)$ in rule bodies, which are not allowed in the framework presented in $[80]$. 
In [35] it is shown that, when a total ordering is used in the lattice, the semantics of [80] correspond to the semantics obtained through the fixpoint definition used in this paper.

When the ordering used is not total, however, this equivalence is no longer valid. For example, consider the lattice $(\mathbb{B} \times \mathbb{B}, \leq)$ such that $(1,1)$ is the top element of the lattice, $(0,0)$ is the bottom element and $(0,0) \leq(0,1) \leq(1,1)$ and $(0,0) \leq(1,0) \leq(1,1)$. Now consider an AFASP program $P$, with $\mathcal{A}_{P}(\rho)=\inf \{\rho(r) \mid r \in P\}$, over this lattice:

$$
\begin{aligned}
& r_{1}: a \leftarrow(1,0) \\
& r_{2}: a \leftarrow(0,1)
\end{aligned}
$$

According to the property of residual implicators that $x \rightarrow y=1$ iff $x \leq y$, any interpretation $I$ of $P$ that satisfies rule $r_{1}$ to the degree $(1,1)$ must obey $I(a) \geq(1,0)$. Likewise any interpretation $I$ that satisfies $r_{2}$ to the degree $(1,1)$ must obey $I(a) \geq$ $(0,1)$. Hence the only 1 -model of $P$ is $I=\left\{a^{(1,1)}\right\}$. However, according to rule (ii) above $\{a\}$ is an unfounded set, which means that under the unfounded semantics $I$ is not an answer set of $P$. On the other hand, $I=\Pi_{P^{I}, \rho_{I}}^{*}$, and thus $I$ is an answer set of $P$ according to the fixpoint semantics. If we consider rules as constraints that need to be fulfilled, the fixpoint semantics correspond better to our intuition.

\subsection{Valued Constraint Satisfaction Problems}

A classical constraint satisfaction problem (CSP) consists of a set of variables $X=$ $\left\{x_{1}, \ldots, x_{n}\right\}$, a set of finite domains $D=\left\{d_{1}, \ldots, d_{n}\right\}$ such that variable $x_{i}$ ranges over domain $d_{i}$, and a set of constraints $C$ of the form $c=\left(X_{c}, R_{c}\right)$ such that $X_{c} \subseteq X$ is a set of variables and $R_{c}$ is a relation between the variables in $X_{c}$. In Valued Constraint Satisfaction Problems (VCSPs) [68], a CSP is augmented with a cost function $\varphi$, which associates a cost to every constraint. A solution to a VCSP is then an assignment of values to the variables in $X$ such that the aggregated cost of all violated constraints is minimal. Typically, costs are represented as real numbers, and the maximum or sum is used to aggregate.

In the crisp case it has been noted that answer set programming can be used for solving constraint satisfaction problems $[59,65]$. The idea is to write an answer set program containing generate rules ${ }^{4}$ that generate possible assignments of values to each of the variables, and constraints which remove those assignments that violate any constraints. In this way the resulting answer set program models a constraint satisfaction problem in the sense that answer sets of the program correspond to the solutions of the problem under consideration. It should come as no surprise that the AFASP framework can likewise be used for modeling VCSPs, as VCSPs can be seen as CSPs with an added aggregation operator. Basically, a VCSP corresponds to an AFASP program that only uses choice rules (which are the fuzzy equivalent of generate rules, i.e. rules assigning a random truth value to a certain atom) and constraints. Hard constraints correspond to rules that are required to be greater than 1 in the aggregator, whereas soft contstraints are rules whose valuation in the aggregator can be lower than 1 , such as rules aggregated using the infimum. An example of the use of this paradigm

\footnotetext{
4 These are rules involving cyclic negation such as the ASP program $\{a \leftarrow$ not $b . b \leftarrow$ not $a\}$. The answer sets of this program are $\{a\}$ and $\{b\}$, hence this program allows us to choose one of two options $a$ and $b$ in a solution of the modeled problem.
} 
is the fuzzy graph coloring program introduced in Section 1, where we model the constraint satisfaction problem of coloring a graph with continuous colors, given some soft and hard constraints.

\subsection{Answer Set Optimization}

In $[6,7]$, a framework for answer set optimization is proposed. The basic idea is that one can state preference rules which are combined to define an ordering over answer sets. For example, if we have a program that generates a class room schedule, this framework allows to state that if teacher John is teaching Math, we prefer John to also teach Physics as follows:

$$
\text { teaches(John, Physics) : } 0>\text { teaches(Mark, Physics) }: 1 \leftarrow \text { teaches(John, Math) }
$$

A rule is of the general form $C_{1}: p_{1}>\ldots>C_{k}: p_{k} \leftarrow a_{1}, \ldots, a_{n}$, not $b_{1}, \ldots$, not $b_{m}$, where $C_{i}: p_{i}$ encodes that the penalty associated with the rule is $p_{i}$ if $i$ is the lowest index for which the atom set $C_{i}$ is true. However, the penalty of the rule is only taken into account if the conditions on the left hand side, expressed as a conjunction of atoms, are true. Different rules can then be combined using strategies, which encode importance among these preference rules. Formally, [6] defines a Preference Description Language $P D L$ in which one can for example write that answer sets should be ordered using the Pareto ordering on rules $r_{1}$ and $r_{2}$ as (pareto $r_{1}, r_{2}$ ). Many other complex (combinations of) orderings can be written in this language, such as (lex (pareto $\left.\left.r_{1}, r_{2}\right), r_{3}\right)$ which denotes that two answer sets first need to be compared using the Pareto ordering on rules $r_{1}$ and $r_{2}$; if they are Pareto-equal, then one must try to discriminate between them on the basis of rule $r_{3}$.

It is clear that the ideas of this approach and the one we proposed in this paper are very similar. In [36] we showed how this framework could be generalized to AFASP, using an appropriate aggregator. For a practical implementation of AFASP it seems interesting to adopt the same strategy of having a fixed language for specifying the aggregator. For example, we could then write an aggregator defining a lexicographical ordering over the program rules $r_{1}, r_{2}, r_{3}$ as $\left(\right.$ lex $\left.r_{1}, r_{2}, r_{3}\right)$.

\subsection{Embedding AFASP in FASP}

One may wonder whether AFASP can be trivially embedded in FASP. Indeed, at first sight it seems that AFASP programs can be simulated by using a new rule aggr $\leftarrow$ $f\left(a_{1}, \ldots, a_{n}\right)$, where $a_{i}$, for $i \in\{1, \ldots, n\}$, is a new atom that corresponds to the value of rule $r_{i}$. However, this intuitive embedding changes the semantics of an AFASP program. For example, consider the following AFASP program $P$ :

$$
\begin{aligned}
& r_{1}: a \leftarrow_{m} \sim_{l} b \\
& r_{2}: b \leftarrow_{m} 0.7
\end{aligned}
$$


We assume that the aggregator is $\mathcal{A}_{P}(\rho)=\min \left(\rho\left(r_{1}\right), \rho\left(r_{2}\right)\right)$. Intuitively one may expect that $P$ can be simulated using the following program $P^{\prime}$ :

$$
\begin{aligned}
r_{1}: & a & \leftarrow \sim_{l} b \\
r_{2}: & b & \leftarrow 0.7 \\
r_{a}: & r_{1}^{\prime} & \leftarrow\left(\sim_{l} b \rightarrow_{m} a\right) \\
r_{b}: & r_{2}^{\prime} & \leftarrow\left(0.7 \rightarrow_{m} b\right) \\
r_{a g g r} & : \text { aggr } & \leftarrow \min \left(r_{1}^{\prime}, r_{2}^{\prime}\right)
\end{aligned}
$$

Now consider the 0.5 -answer set $A=\left\{a^{0.5}, b^{0.5}\right\}$ of $P$. The corresponding interpretation $A^{\prime}=\left\{a^{0.5}, b^{0.5}, r_{1}^{\prime 1}, r_{2}^{\prime 0.5}, a g g r^{0.5}\right\}$ is not a model of $P^{\prime}$ because rule $r_{2}$ is not satisfied to the degree 1 . Therefore it is also not an answer set of $P^{\prime}$, meaning this translation does not preserve the semantics.

The correct translation is provided by the following program $P^{\prime \prime}$ :

$$
\begin{array}{rlrl}
r_{1}: & a & \leftarrow \sim_{l} b \wedge_{m} r_{1}^{\prime} \\
r_{2}: & b & \leftarrow 0.7 \wedge_{m} r_{2}^{\prime} \\
r_{a}: & r_{1}^{\prime} & \leftarrow\left(\sim_{l} b \rightarrow_{m}\left(\sim_{l} \operatorname{not}_{a}\right)\right) \\
r_{b} & : \quad r_{2}^{\prime} & \leftarrow\left(0.7 \rightarrow_{m}\left(\sim_{l} \operatorname{not}_{b}\right)\right) \\
r_{n a}: \operatorname{not}_{a} & \leftarrow \sim_{l} a \\
r_{n b}: \operatorname{not}_{b} & \leftarrow \sim_{l} b \\
r_{a g g r}: & : \text { aggr } & \leftarrow \min \left(r_{1}^{\prime}, r_{2}^{\prime}\right)
\end{array}
$$

One can easily verify that $A^{\prime \prime}=A^{\prime} \cup\left\{n o t_{a}^{0.5}, n o t_{b}^{0.5}\right\}$ is an answer set of $P^{\prime \prime}$. Furthermore it can be shown that there is a bijection between the answer sets of $P$ and $P^{\prime \prime}$. However, as is evident from the above, this translation is not very intuitive. Moreover it only works for aggregators that map rule interpretations to the truth lattice of the program and not for aggregators mapping rule interpretations to more interesting preorders. Hence, even though (some) AFASP programs can be translated to FASP programs, the study and use of AFASP on its own is still worthwhile.

\section{Conclusions}

FASP can be used for modeling continuous optimization problems. Due to the continuity, it is natural to have some notion of approximate answer sets, which correspond to models that satisfy some of the rules only partially. In current approaches, however, the idea of partial rule satisfaction is implemented in a rather limited way: users are required to state to what extent each rule should be satisfied.

In this paper, we have introduced a more flexible method for handling partial rule satisfaction, which is based on aggregation operators that combine the degrees to which each of the rules are satisfied to a single value from a preordered set. In this sense, in our approach, the weights that are assigned to rules are variable, and the aggregator determines which combinations of rule weights are most desirable. Our work extends a previous proposal for using aggregators for FASP programs in two ways. First, the preorder determined by the aggregator is decoupled from the truth lattice considered in the program. This makes it possible to express preference among solutions of problems in a more flexible way. Second, we have developed a fixpoint semantics, in contrast to existing work which is based on unfounded semantics. This has the advantage that we are no longer restricted to using t-norms in rule bodies and more closely shows the 
links between the other FASP approaches. Furthermore it solves a problem with the previous proposal when generalizing from $([0,1], \leq)$ to arbitrary truth lattices.

We have also illustrated our approach on two examples: continuous graph coloring and the reviewer assignment problem. By means of these examples we have illustrated the practical usefulness of our framework.

The implementation of our framework depends on the aggregators and body functions that are used. In [34] we have shown that when the aggregator ranges over the truth lattice of the program and the body functions are t-norms, we can solve AFASP programs using fuzzy SAT solvers. When the leximin or discrimin is used, we can use techniques from constraint satisfaction solving such as [20]. Last, for programs with a linear aggregator and linear functions in rule bodies we can use multi-level mixed integer programming, as mentioned in [69]. In future research we plan to investigate these implementation methods in more detail.

Acknowledgements Jeroen Janssen is funded by a research project from the Flemish Fund for Scientific Research (FWO-Vlaanderen). Steven Schockaert is a post-doctoral fellow of the Flemish Fund for Scientific Research (FWO-Vlaanderen).

\section{A Proofs}

\section{A.1 Proofs of Section 3}

Proof (Proof of Proposition 1) Suppose $r: a \leftarrow \alpha$ is defined over a lattice $\mathcal{L}$, then:

$$
\begin{aligned}
& I_{s}(r, c)=\quad\left\langle\text { Def. } I_{s}(r, c)\right\rangle \quad \inf \{y \in \mathcal{L} \mid I(\alpha) \rightarrow y \geq c\} \\
& =\langle\text { Residuation principle }\rangle \inf \left\{y \in \mathcal{L} \mid y \geq I(\alpha) \wedge_{r} c\right\} \\
& =\langle\text { See below }\rangle \quad I(\alpha) \wedge_{r} c
\end{aligned}
$$

The last step follows from the fact that $I(\alpha) \wedge_{r} c$ is an element of $\mathcal{L}$ and is a lower bound of $\left\{y \in \mathcal{L} \mid y \geq I(\alpha) \wedge_{r} c\right\}$.

Proof (Proof of Proposition 3)

$$
\begin{aligned}
& \Pi_{P, \rho_{1}}(I)(a)=\left\langle\text { Def. } \Pi_{P, \rho_{1}}\right\rangle \quad \sup \left\{I_{s}\left(\rho_{1}, r\right) \mid r \in P_{a}\right\} \\
& =\langle\text { Proposition 1 }\rangle \sup \left\{I\left(r_{b}\right) \wedge_{r} \rho_{1}(r) \mid r \in P_{a}\right\} \\
& \leq\left\langle\text { Monot. t-norm〉 } \sup \left\{I\left(r_{b}\right) \wedge_{r} \rho_{2}(r) \mid r \in P_{a}\right\}\right. \\
& =\langle\text { Proposition } 1\rangle \sup \left\{I_{s}\left(\rho_{2}, r\right) \mid r \in P_{a}\right\} \\
& =\left\langle\text { Def. } \Pi_{P, \rho_{2}}\right\rangle \quad \Pi_{P, \rho_{2}}(I)(a)
\end{aligned}
$$

Proof (Proof of Proposition 4) It is straightforward to show, using transfinite induction and Propositions 2 and 3 , that, for any ordinal $i, J_{i}^{1} \leq J_{i}^{2}$, where $\mathcal{S}\left\langle P, \rho_{1}\right\rangle=\left\langle J_{i}^{1}\right| i$ an ordinal $\rangle$ and $\mathcal{S}\left\langle P, \rho_{2}\right\rangle=\left\langle J_{i}^{2}\right| i$ an ordinal $\rangle$.

Indeed: $J_{0}^{1}=J_{0}^{2}=\emptyset$. Propositions 2 and 3 then ensure that, for a successor ordinal $i$, $J_{i}^{1}=\Pi_{P, \rho_{1}}\left(J_{i-1}^{1}\right) \leq \Pi_{P, \rho_{2}}\left(J_{i-1}^{2}\right)=J_{i}^{2}$ follows from $\rho_{1} \leq \rho_{2}$ and the induction hypothesis. For a limit ordinal $i, J_{i}^{1}=\bigcup_{j<i} J_{j}^{1} \leq \bigcup_{j<i} J_{j}^{2}=J_{i}^{2}$ is immediate from the induction hypothesis.

Proof (Proof of Proposition 5) Since $P$ is constraint-free, we know that $\mathcal{R}_{P}=\bigcup_{a \in \mathcal{B}_{P}} P_{a}$. Using this property we obtain the stated as follows:

$$
\begin{array}{lll} 
& M=\Pi_{P, \rho}(M) \\
\equiv \quad\left\langle\text { Def. } \Pi_{P, \rho}(M)\right\rangle & \forall a \in \mathcal{B}_{P} \cdot M(a)=\sup \left\{M_{s}(r, \rho(r)) \mid r \in P_{a}\right\} \\
\Rightarrow \quad\langle\text { sup is upper bound }\rangle & \forall a \in \mathcal{B}_{P} \cdot \forall r \in P_{a} \cdot M(a) \geq M_{s}(r, \rho(r)) \\
\equiv\left\langle\text { Prop. } 1, \mathcal{R}_{P}=\cup_{a \in \mathcal{B}_{P}} P_{a}\right\rangle & \forall r \in \mathcal{R}_{P} \cdot M\left(r_{h}\right) \geq M\left(r_{b}\right) \wedge_{r} \rho(r) \\
\equiv \quad\langle\text { Residuation principle }\rangle & \forall r \in \mathcal{R}_{P} \cdot M\left(r_{b}\right) \rightarrow M\left(r_{h}\right) \geq \rho(r) \\
\equiv \quad \text { DDef. } \rho \text {-rule model }\rangle & M \text { is a } \rho \text {-rule model of } P
\end{array}
$$


Proof (Proof of Proposition 6) Since $P$ is constraint-free, we know that $\mathcal{R}_{P}=\bigcup_{a \in \mathcal{B}_{P}} P_{a}$. Now, suppose that $M$ is a minimal $\rho$-rule model of $P$ and not a fixpoint of $\Pi_{P, \rho}$. Then we first show that $\forall a \in \mathcal{B}_{P} \cdot M(a) \geq \sup _{r \in P_{a}} M_{s}(r, \rho(r))$ as follows:

$$
\begin{aligned}
& \quad M \text { is a } \rho \text {-rule model of } P \\
& \equiv \quad\langle\text { Def. } \rho \text {-rule model }\rangle \quad \forall r \in \mathcal{R}_{P} \cdot M\left(r_{b}\right) \rightarrow_{r} M\left(r_{h}\right) \geq \rho(r) \\
& \equiv\left\langle\text { Residuation principle } \quad \forall r \in \mathcal{R}_{P} \cdot M\left(r_{h}\right) \geq M\left(r_{b}\right) \wedge_{r} \rho(r)\right. \\
& \equiv\left\langle\text { Prop. } 1, \mathcal{R}_{P}=\cup_{a \in \mathcal{B}_{P}} P_{a}\right\rangle \quad \forall a \in \mathcal{B}_{P} \cdot \forall r \in P_{a} \cdot M(a) \geq M_{s}(r, \rho(r)) \\
& \equiv\langle\text { Def. upper bound, } \sup \rangle \quad \forall a \in \mathcal{B}_{P} \cdot M(a) \geq \sup _{r \in P_{a}} M_{s}(r, \rho(r))
\end{aligned}
$$

As $M$ is not a fixpoint of $\Pi_{P, \rho}$, for some $a \in \mathcal{B}_{P}$ it must hold that $M(a)>\sup _{r \in P_{a}} M_{s}(r, \rho(r))$. Consider then the interpretation $M^{\prime}$ defined as

$$
M^{\prime}(x)= \begin{cases}M(x) & \text { if } x \neq a \\ \sup _{r \in P_{a}} M_{s}(r, \rho(r)) & \text { if } x=a\end{cases}
$$

Clearly $M^{\prime}<M$. We will show that $M^{\prime}$ is a $\rho$-rule model, leading to a contradiction with the minimality of $M$. For any $x \in\left(\mathcal{B}_{P} \backslash\{a\}\right)$ we show that

$$
\forall r \in P_{x} \cdot M^{\prime}\left(r_{b}\right) \rightarrow M^{\prime}(x) \geq \rho(r)
$$

Indeed:

$$
\begin{aligned}
M^{\prime}<M & \Rightarrow\langle\text { Body is incr. function }\rangle & M^{\prime}\left(r_{b}\right) \leq M\left(r_{b}\right) \\
& \Rightarrow\langle\text { Anti-monoton. } \rightarrow\rangle & M^{\prime}\left(r_{b}\right) \rightarrow M(x) \geq M\left(r_{b}\right) \rightarrow M(x) \\
& \Rightarrow\langle M \text { is } \rho \text {-rule model }\rangle & M^{\prime}\left(r_{b}\right) \rightarrow M(x) \geq \rho(r) \\
& \equiv\left\langle\text { Def. } M^{\prime}, r_{h}=x \neq a\right\rangle & M^{\prime}\left(r_{b}\right) \rightarrow M^{\prime}(x) \geq \rho(r)
\end{aligned}
$$

For $a$ we show that

$$
\forall r \in P_{a} \cdot M^{\prime}\left(r_{b}\right) \rightarrow M^{\prime}(a) \geq \rho(r)
$$

as follows:

$$
\begin{array}{ll} 
& M^{\prime}(a)=\sup _{r \in P_{a}} M_{s}(r, \rho(r)) \\
\Rightarrow \quad \text { s sup is upper bound }\rangle & \forall r \in P_{a} \cdot M^{\prime}(a) \geq M_{s}(r, \rho(r)) \\
\equiv \quad \text { (Prop. 1 } & \forall r \in P_{a} \cdot M^{\prime}(a) \geq M\left(r_{b}\right) \wedge_{r} \rho(r) \\
\Rightarrow\left\langle M^{\prime}<M, \text { Monot. } \wedge_{r},(*)\right\rangle & \forall r \in P_{a} \cdot M^{\prime}(a) \geq M^{\prime}\left(r_{b}\right) \wedge_{r} \rho(r) \\
\equiv \quad\langle\text { Residuation principle }\rangle & \forall r \in P_{a} \cdot M^{\prime}\left(r_{b}\right) \rightarrow M^{\prime}(a) \geq \rho(r)
\end{array}
$$

The $(*)$ justification is that $r_{b}$ contains an increasing function. By combining (12) and (13), we obtain that $M^{\prime}$ is a $\rho$-rule model, contradicting the assumption that $M$ is a minimal $\rho$-rule model. Hence $M$ must be a fixpoint of $\Pi_{P, \rho}$.

Proof (Proof of Proposition 7) First, we show that $\Pi_{P, \rho}^{*}$ is a minimal $\rho$-rule model of $P$. Due to Proposition 5 we know that $\Pi_{P, \rho}^{*}$ must be a $\rho$-rule model of $P$. Suppose $M$ is a $\rho$-rule model such that $M \leq \Pi_{P, \rho}^{*}$. Without loss of generalisation, we can assume that $M$ is a minimal $\rho$-rule model. Now from Proposition 6 we know that $M$ must be a fixpoint of $\Pi_{P, \rho}$ and hence, as $\Pi_{P, \rho}^{*}$ is the least fixpoint of $\Pi_{P, \rho}$, that $M=\Pi_{P, \rho}^{*}$. Thus $\Pi_{P, \rho}^{*}$ is a minimal $\rho$-rule model of $P$.

Second, we show that no other minimal $\rho$-rule models of $P$ exist. Suppose $M$ is a minimal $\rho$-rule model of $P$. From Proposition 6 we know that $M$ must be a fixpoint of $\Pi_{P, \rho}$ and hence $\Pi_{P, \rho}^{*} \leq M$ as $\Pi_{P, \rho}^{*}$ is the least fixpoint of $\Pi_{P, \rho}$. From this it follows that $M=\Pi_{P, \rho}^{*}$.

Proof (Proof of Corollary 1) Follows immediately from Proposition 7.

Lemma 1 Let $P$ be an AFASP program and $I$ an interpretation of this program, then for each $a \in \mathcal{B}_{P}$ it holds that $I(a)$ is an upper bound of the set $\left\{I_{s}\left(r, \rho_{I}(r)\right) \mid r \in P_{a}\right\}$.

Proof We show that for any $a \in \mathcal{B}_{P}$ and $r \in P_{a}$ it holds that $I(a) \geq I_{s}\left(r, \rho_{I}(r)\right)$, from which the stated readily follows.

$$
\begin{array}{rlll}
I_{s}\left(r, \rho_{I}(r)\right) & =\left\langle\text { Def. } I_{s}\left(r, \rho_{I}(r)\right)\right\rangle & \inf \left\{y \in \mathcal{L}_{P} \mid I\left(r_{b}\right) \rightarrow y \geq \rho_{I}(r)\right\} \\
& =\quad\left\langle\text { Def. } \rho_{I}\right\rangle & \inf \left\{y \in \mathcal{L}_{P} \mid I\left(r_{b}\right) \rightarrow y \geq I\left(r_{b}\right) \rightarrow I(a)\right\} \\
& \leq \quad\langle\text { Def. inf }\rangle & I(a) &
\end{array}
$$


Proof (Proof of Proposition 8) First, note that from Proposition 5 we can immediately see that $\rho \leq \rho_{M}$ as $M$ is a fixpoint of $\Pi_{P, \rho}$. Second, we show that $\Pi_{P, \rho_{M}}(M)=M$ :

$$
\begin{array}{rlll}
M=\Pi_{P, \rho}^{*} & \Rightarrow\langle\text { Def. fixpoint }\rangle & \forall a \in \mathcal{B}_{P} \cdot M(a)=\Pi_{P, \rho}(M)(a) \\
& \Rightarrow\left\langle\rho \leq \rho_{M}, \text { Prop. } 3\right\rangle & \forall a \in \mathcal{B}_{P} \cdot M(a) \leq \Pi_{P, \rho_{M}}(M)(a) \\
& \equiv\left\langle\text { Def. } \Pi_{P, \rho_{M}}\right\rangle & \forall a \in \mathcal{B}_{P} \cdot M(a) \leq \sup _{r \in P_{a}} M_{s}\left(r, \rho_{M}(r)\right) \\
& \Rightarrow \quad\langle\text { Lemma } 1\rangle & \forall a \in \mathcal{B}_{P} \cdot M(a)=\sup _{r \in P_{a}} M_{s}\left(r, \rho_{M}(r)\right) \\
& \equiv \quad\left\langle\text { Def. } \Pi_{P, \rho_{M}}\right\rangle & \forall a \in \mathcal{B}_{P} \cdot M(a)=\Pi_{P, \rho_{M}}(M)(a)
\end{array}
$$

By Proposition 4 and the fact that $\rho \leq \rho_{M}$ we obtain that $\Pi_{P, \rho}^{*} \leq \Pi_{P, \rho_{M}}^{*}$ and thus by definition of $M$ that $M \leq \Pi_{P, \rho_{M}}^{*}$. As we have shown that $M$ is a fixpoint of $\Pi_{P, \rho_{M}}$, and thus $\Pi_{P, \rho_{M}}^{*} \leq M$, this means that $M=\Pi_{P, \rho_{M}}^{*}$.

Proof (Proof of Proposition 9) Due to Proposition 8 we already know that $M=\Pi_{P, \rho_{M}}^{*}$. We thus only need to show that $\mathcal{A}_{P}\left(\rho_{M}\right) \geq \mathcal{A}_{P}(\rho)$. From Proposition 5 , we know that $M$ is a $\rho$-rule model, i.e. $\forall r \in \mathcal{R}_{P} \cdot \rho_{M}(r) \geq \rho(r)$. This implies $\mathcal{A}_{P}\left(\rho_{M}\right) \geq \mathcal{A}_{P}(\rho)$ because $\mathcal{A}_{P}$ is increasing.

Proof (Proof of Corollary 2) First, suppose $M$ is a $k$-answer set of a simple AFASP program $P$. By definition of $k$-answer sets it must then hold that $\mathcal{A}_{P}\left(\rho_{M}\right) \geq k$ and that $M=\Pi_{P, \rho_{M}}^{*}$, hence some rule interpretation $\rho$ exists such that $M=\Pi_{P, \rho}^{*}$ and $\mathcal{A}_{P}(\rho) \geq k$.

Second, suppose there is some rule interpretation $\rho$ for which $\mathcal{A}_{P}(\rho) \geq k$ and $M=\Pi_{P, \rho}^{*}$. From Proposition 9 we then know that $M$ is a $\mathcal{A}_{P}(\rho)$-answer set of $P$, from which we know that $\mathcal{A}_{P}\left(\rho_{M}\right) \geq \mathcal{A}_{P}(\rho)$ and hence, as $\mathcal{A}_{P}(\rho) \geq k$, it follows that $\mathcal{A}_{P}\left(\rho_{M}\right) \geq k$. Thus $M$ is a $k$-answer set.

Proof (Proof of Corollary 3) Let $P$ be a simple AFASP program. The desired answer set is obtained by applying Proposition 8 to the rule interpretation $\rho_{\top}$.

Proof (Proof of Proposition 10) From definitions 11 and 12, it immediately follows that $\mathcal{A}_{P}\left(\rho_{M}\right)=\mathcal{A}_{P^{M}}\left(\rho_{M}\right) \geq k$.

Proof (Proof of Proposition 11) Let $M$ be a $k$-answer set of $P$, then by definition of answer sets we know that $M=\Pi_{P}^{*}, \rho_{M}$. We will show by contradiction that no $M^{\prime} \subset M$ can exist such that $M^{\prime}$ is a $\rho_{M}$-rule model of $P$. Suppose $M^{\prime} \subset M$ such that $M^{\prime}$ is a $\rho_{M}$-rule model of $P$. From this it follows that for any rule $r \in P$ we have $M^{\prime}\left(r_{b}\right) \geq M^{\prime}\left(r_{b}^{M}\right)$ as only the arguments in which the body function is decreasing are replaced by their value in $M$. We can then proceed as follows:

$$
\begin{array}{lll} 
& M^{\prime} \text { is a } \rho_{M} \text {-rule model of } P \\
\equiv\left\langle\text { Def. } \rho_{M} \text {-rule model }\right\rangle & \forall r \in \mathcal{R}_{P} \cdot M^{\prime}\left(r_{b}\right) \rightarrow M^{\prime}\left(r_{h}\right) \geq \rho_{M}(r) \\
\equiv \quad\langle\text { Property reduct }\rangle & \forall r \in \mathcal{R}_{P} \cdot M^{\prime}\left(r_{b}\right) \rightarrow M^{\prime}\left(r_{h}\right) \geq \rho_{M}\left(r^{M}\right) \\
\Rightarrow\left\langle M^{\prime}\left(r_{b}\right) \geq M^{\prime}\left(r_{b}^{M}\right)\right\rangle & \forall r \in \mathcal{R}_{P} \cdot M^{\prime}\left(r_{b}^{M}\right) \rightarrow M^{\prime}\left(r_{h}\right) \geq \rho_{M}\left(r^{M}\right) \\
\equiv\left\langle r_{h} \in \mathcal{B}_{P}, \text { Def. reduct }\right\rangle & \forall r \in \mathcal{R}_{P} \cdot M^{\prime}\left(r_{b}^{M}\right) \rightarrow M^{\prime}\left(r_{h}^{M}\right) \geq \rho_{M}\left(r^{M}\right) \\
\equiv \quad\left\langle\text { Def. } P^{M}\right\rangle & \forall r \in \mathcal{R}_{P} \cdot M^{\prime}\left(r_{b}\right) \rightarrow M^{\prime}\left(r_{h}\right) \geq \rho_{M}(r) \\
\equiv\left\langle\text { Def. } \rho_{M} \text {-rule model }\right\rangle & M^{\prime} \text { is a } \rho_{M} \text {-rule model of } P^{M}
\end{array}
$$

From Proposition 7 and the fact that $M=\Pi_{P^{M}, \rho_{M}}$ we know that $M$ is the unique minimal $\rho_{M}$-rule model of $P^{M}$, leading to a contradiction with the fact that $M^{\prime}$ is a $\rho_{M}$-rule model of $P^{M}$ and $M^{\prime} \subset M$.

Lemma 2 Let $P$ be an AFASP program, then an interpretation $I$ is a fixpoint of $\Pi_{P, \rho}$ iff it is a fixpoint of $\Pi_{P^{I}, \rho}$.

Proof First, remark that for any expression $\alpha$ and interpretation $I$ we have that $I(\alpha)=I\left(\alpha^{I}\right)$. Then we proceed as follows.

$$
\begin{array}{ll} 
& I=\Pi_{P, \rho}(I) \\
\equiv\langle\text { Equality of functions }\rangle & \forall a \in \mathcal{B}_{P} \cdot I(a)=\Pi_{P, \rho}(I)(a) \\
\equiv \quad\left\langle\text { Def. } \Pi_{P, \rho}\right\rangle & \forall a \in \mathcal{B}_{P} \cdot I(a)=\sup _{r \in P_{a}} I_{s}(r, \rho(r)) \\
\equiv \quad\langle\text { Prop. } 1\rangle & \forall a \in \mathcal{B}_{P} \cdot I(a)=\sup _{r \in P_{a}} I\left(r_{b}\right) \wedge_{r} \rho(r) \\
\equiv \quad\left\langle I(\alpha)=I\left(\alpha^{I}\right)\right\rangle & \forall a \in \mathcal{B}_{P} \cdot I(a)=\sup _{r \in P_{a}} I\left(r_{b}^{I}\right) \wedge_{r} \rho(r) \\
\equiv\left\langle\text { Def. } \Pi_{P, \rho}, \text { Def. } P^{I}\right\rangle & \forall a \in \mathcal{B}_{P} \cdot I(a)=\Pi_{P^{I}, \rho}(I)(a) \\
\equiv & I=\Pi_{P^{I}, \rho}(I)
\end{array}
$$


Proof (Proof of Proposition 12) From Lemma 2, we know that any answer set $M$ must be a fixpoint of $\Pi_{P, \rho_{M}}$. Suppose $M$ is not a minimal fixpoint and thus, that some $N$ exists, $N \subset M$, such that $N$ is a fixpoint of $\Pi_{P, \rho_{M}}$. We will show that any such $N$ is also a $\rho_{M}$-rule model of $P^{M}$, leading to $N \supseteq M$ due to Proposition 7 , a contradiction.

First, as $N \subset \bar{M}$ and since the reduct only substitutes negative subexpressions for their corresponding values, it follows that, for any expression $\alpha$ :

$$
N\left(\alpha^{N}\right) \geq N\left(\alpha^{M}\right)
$$

and thus, since $N(\alpha)=N\left(\alpha^{N}\right)$ that

$$
N(\alpha) \geq N\left(\alpha^{M}\right)
$$

We can now show that $N$ is a $\rho_{M}$-rule model of $P^{M}$ as follows.

$$
\begin{array}{cll} 
& N=\Pi_{P, \rho_{M}}(N) \\
\Rightarrow & \langle\text { Prop. } 5\rangle & \forall r \in \mathcal{R}_{P} \cdot N\left(r_{b}\right) \rightarrow N\left(r_{h}\right) \geq \rho_{M}(r) \\
\Rightarrow\left\langle\text { Anti-monot. } \rightarrow, N\left(r_{b}\right) \geq N\left(r_{b}^{M}\right)\right\rangle & \forall r \in \mathcal{R}_{P} \cdot N\left(r_{b}^{M}\right) \rightarrow N\left(r_{h}\right) \geq \rho_{M}(r) \\
\equiv \quad & \forall r \in \mathcal{R}_{P} \cdot N\left(r_{b}\right) \rightarrow N\left(r_{h}\right) \geq \rho_{M}(r) \\
\equiv \quad & & \forall r \in \mathcal{R}_{P}^{M} \cdot N(r) \geq \rho_{M}(r)
\end{array}
$$

\section{A.2 Proofs of Section 5}

Proof (Proof of Proposition 13) First, remark that there is only a single rule interpretation $\rho$ such that $\forall r \in P_{\text {weight }} \cdot \rho(r)=1$ holds, viz. $\rho \top$. As $A$ is only a 1 -answer set of $P_{w e i g h t}$ iff $A=\Pi_{P, \rho}^{*}$ for some rule interpretation satisfying $\forall r \in P_{\text {weight }} \cdot \rho(r)=1$ due to Corollary 2, it must hold that there is a unique 1 -answer set $A$ of $P_{\text {weight }}$ where $A=\Pi_{P, \rho \top}^{*}$. We can now show that $\Pi_{P}=\Pi_{P, \rho \top}$, leading to the stated equivalence, as follows. Suppose $I$ is some interpretation of $P$ (and hence also of $P_{\text {weight }}$ ) and $l \in \mathcal{B}_{P}$ (and hence also $l \in \mathcal{B}_{P_{\text {weight }}}$ ), then:

$$
\begin{aligned}
& \Pi_{P}(I)(l)=\left\langle\text { Def. } \Pi_{P}\right\rangle \quad \sup \left\{I\left(r_{b} \wedge_{r} r_{w}\right) \mid r \in P_{l}\right\} \\
& =\left\langle\text { Def. } P_{\text {weight }}\right\rangle \quad \sup \left\{I\left(r_{b}\right) \mid r \in\left(P_{\text {weight }}\right)_{l}\right\} \\
& =\langle\text { Def. } \rho \top\rangle \quad \sup \left\{I\left(r_{b} \wedge_{r} \rho_{\top}(r)\right) \mid r \in\left(P_{\text {weight }}\right)_{l}\right\}
\end{aligned}
$$

From this, the stated readily follows.

Proof (Proof of Proposition 14) The proof follows easily from Proposition 13 and the fact that the Gelfond-Lifschitz reduct introduced in [53] is a special case of our reduct.

\section{References}

1. Fundamentals of Uncertainty Calculi with Applications to Fuzzy Inference. Kluwer Academics Publishers (1995)

2. Alsinet, T., Godo, L., Sandri, S.: Two formalisms of extended possibilistic logic programming with context-dependent fuzzy unification: A comparative description. Electronic Notes in Theoretical Computer Science 66(5), 1 - 21 (2002)

3. Balog, K., de Rijcke, M.: Determining expert profiles (with an application to expert finding). In: Proceedings of the International Joint Conference on Artificial Intelligence (IJCAI07), pp. 2657-2662 (2007)

4. Baral, C.: Knowledge Representation, Reasoning and Declarative Problem Solving. Cambridge University Press (2003)

5. Bauters, K., Schockaert, S., De Cock, M., Vermeir, D.: Possibilistic answer set programming revisited. In: Proceedings of the 26th Conference on Uncertainty in Artificial Intelligence (UAI) (2010)

6. Brewka, G.: Complex preferences for answer set optimization. In: D. Dubois, C.A. Welty, M.A. Williams (eds.) KR, pp. 213-223 (2004) 
7. Brewka, G., Niemelä, I., Truszczyński, M.: Answer set optimization. In: Proceedings of the 18th International Joint Conference on Artificial Intelligence, pp. 867-872 (2003)

8. Cao, T.H.: Annotated fuzzy logic programs. Fuzzy Sets \& Systems 113(2), 277-298 (2000)

9. Choquet, G.: Theory of capacities. Annales de l'Institut Fourier 5, 131-295 (1954)

10. Damásio, C.V., Medina, J., Ojeda-Aciego, M.: Sorted multi-adjoint logic programs: termination results and applications. In: Proceedings of the 9th European Conference on Logics in Artificial Intelligence (JELIA'04), pp. 260-273 (2004)

11. Damásio, C.V., Medina, J., Ojeda-Aciego, M.: Termination of logic programs with imperfect information: applications and query procedure. Journal of Applied Logic 5(3), 435-458 (2007)

12. Damásio, C.V., Pereira, L.M.: Hybrid probabilistic logic programs as residuated logic programs. In: Proceedings of the 7th European Workshop on Logics in Artificial Intelligence (JELIA'00), pp. 57-72 (2000)

13. Damásio, C.V., Pereira, L.M.: Antitonic logic programs. In: Proceedings of the 6th International Conference on Logic Programming and Nonmonotonic Reasoning (LPNMR'01), pp. 379-392 (2001)

14. Damásio, C.V., Pereira, L.M.: Monotonic and residuated logic programs. In: Proceedings of the 6th European Conference on Symbolic and Quantitative Approaches to Reasoning with Uncertainty (ECSQARU'01), pp. 748-759 (2001)

15. Damásio, C.V., Pereira, L.M.: Sorted monotonic logic programs and their embeddings. In: Proceedings of Information Processing and Management of Uncertainty (IPMU04), pp. 807-814 (2004)

16. De Cock, M.: A thorough study of linguistic modifiers in fuzzy set theory. Ph.D. thesis, Ghent University (2002)

17. Dekhtyar, A., Subrahmanian, V.S.: Hybrid probabilistic programs. In: Proceedings of the Fourteenth International Conference on Logic Programming (ICLP'97), pp. 391-405 (1997)

18. Detyniecki, M.: Numerical aggregation operators: state of the art. In: Proceedings of the First International Summer School on Aggregation Operators and their Applications (2001)

19. Dubois, D., Fargier, H., Prade, H.: Refinements of the maximin approach to decisionmaking in a fuzzy environment. Fuzzy Sets \& Systems 81(1), 103-122 (1996)

20. Dubois, D., Fortemps, P.: Computing improved optimal solutions to max-min flexible constraint satisfaction problems. European Journal of Operational Research 118, 95-126 (1999)

21. Dubois, D., Lang, J., Prade, H.: Towards possibilistic logic programming. In: Proceedings of the Eigth International Conference on Logic Programming (ICLP'91), pp. 581-595 (1991)

22. Dubois, D., Prade, H.: Weighted minimum and maximun operations in fuzzy sets theory. Information Sciences 39(2), 205-210 (1986)

23. Dubois, D., Prade, H.: Advances in the egalitarist approach to decision-making in a fuzzy environment. In: Y. Yoshida (ed.) Dynamical Aspect in Fuzzy Decision Making, Studies in Fuzziness and Soft Computing, vol. 73, pp. 213-240 (2001)

24. Emden, M.H.v.: Quantitative deduction and its fixpoint theory. Journal of Logic Programming 30(1), 37-53 (1986)

25. Fagin, R., Wimmers, E.L.: A formula for incorporating weights into scoring rules. Theoretical Computer Science 239, 309-338 (1998)

26. Fitting, M.: Bilattices and the semantics of logic programming. Journal of Logic Programming 11(2), 91-116 (1991)

27. Fodor, J.C., Yager, R.R., Rybalov, A.: Structure of uninorms. Int. J. Uncertain. Fuzziness Knowl.-Based Syst. 5(4), 411-427 (1997)

28. Fuhr, N.: Probabilistic datalog: implementing logical information retrieval for advanced applications. Journal of the American Society for Information Science 51(2), 95-110 (2000)

29. Garg, N., Kavitha, T., Kumar, A., Mehlhorn, K., Mestre, J.: Assigning papers to referees (2008)

30. Gelfond, M., Lifschitz, V.: The stable model semantics for logic programming. In: Proceedings of the Fifth International Conference and Symposium on Logic Programming (ICLP/SLP'88), pp. 1081-1086 (1988)

31. Grabisch, M.: k-additive fuzzy measures. In: Proceedings of the 6th international conference on information processing and management of uncertainty in Knowledge-Based systems (IPMU96) (1996) 
32. Hájek, P.: Metamathematics of Fuzzy Logic (Trends in Logic) (2001)

33. Ishizuka, M., Kanai, N.: Prolog-elf incorporating fuzzy logic. In: Proceedings of the 9th international joint conference on Artificial intelligence (IJCAI'85), pp. 701-703 (1985)

34. Janssen, J., Heymans, S., Vermeir, D., De Cock, M.: Compiling fuzzy answer set programs to fuzzy propositional theories. In: Proceedings of the 24th International Conference on Logic Programming (ICLP08) (2008)

35. Janssen, J., Schockaert, S., Vermeir, D., De Cock, M.: Reducing fuzzy answer set programming to model finding in fuzzy logics. Submitted.

36. Janssen, J., Schockaert, S., Vermeir, D., De Cock, M.: Fuzzy answer set programming with literal preferences. In: Proceedings of the Joint 2009 International Fuzzy Systems Association World Congress and 2009 European Society of Fuzzy Logic and Technology Conference (IFSA/EUSFLAT2009), pp. 1347-1352 (2009)

37. Janssen, J., Schockaert, S., Vermeir, D., De Cock, M.: General fuzzy answer set programs. In: V.D. Gesù, S.K. Pal, A. Petrosino (eds.) WILF, Lecture Notes in Computer Science, vol. 5571, pp. 352-359 (2009)

38. Kifer, M., Li, A.: On the semantics of rule-based expert systems with uncertainty. In: Proceedings of the 2nd International Conference on Database Theory (ICDT'88), pp. 102-117 (1988)

39. Kifer, M., Subrahmanian, V.S.: Theory of generalized annotated logic programming and its applications. Journal of Logic Programming 12(3\&4), 335-367 (1992)

40. Lakshmanan, L.V.S.: An epistemic foundation for logic programming with uncertainty. In: Proceedings of the 14th Conference on Foundations of Software Technology and Theoretical Computer Science (FSTTCS'94), pp. 89-100 (1994)

41. Lakshmanan, L.V.S., Sadri, F.: Modeling uncertainty in deductive databases. In: Proceedings of the 5th International Conference on Database and Expert Systems Applications (DEXA'94), pp. 724-733 (1994)

42. Lakshmanan, L.V.S., Sadri, F.: Probabilistic deductive databases. In: Proceedings of the 1994 International Symposium on Logic programming (ILPS'94), pp. 254-268. MIT Press, Cambridge, MA, USA (1994)

43. Lakshmanan, L.V.S., Sadri, F.: Uncertain deductive databases: a hybrid approach. Information Systems 22(9), 483-508 (1997)

44. Lakshmanan, L.V.S., Shiri, N.: A parametric approach to deductive databases with uncertainty. IEEE Transactions on Knowledge and Data Engineering 13(4), 554-570 (2001)

45. Lakshmanan, V.S.: Towards a generalized theory of deductive databases with uncertainty. Ph.D. thesis, Concordia University (1997)

46. Leone, N., Pfeifer, G., Faber, W., Eiter, T., Gottlob, G., Perri, S., Scarcello, F.: The dlv system for knowledge representation and reasoning. ACM Transactions on Computational Logic 7(3), 499-562 (2006)

47. Loyer, Y., Straccia, U.: The well-founded semantics in normal logic programs with uncertainty. In: Proceedings of the 6th International Symposium on Functional and Logic Programming (FLOPS'02), pp. 152-166 (2002)

48. Loyer, Y., Straccia, U.: The approximate well-founded semantics for logic programs with uncertainty. In: Proceedings of the 28th International Symposium on Mathematical Foundations of Computer Science (MFCS'03), pp. 541-550 (2003)

49. Luhandjula, M.K.: Compensatory operators in fuzzy linear programming with multiple objectives. Fuzzy Sets \& Systems 8(3), 245-252 (1982)

50. Lukasiewicz, T.: Probabilistic logic programming. In: Proceedings of the 13th European Conference on Artificial Intelligence (ECAI'98), pp. 388-392 (1998)

51. Lukasiewicz, T.: Many-valued disjunctive logic programs with probabilistic semantics. In: Proceedings of the 5th International Conference on Logic Programming and Nonmonotonic Reasoning (LPNMR'99), pp. 277-289 (1999)

52. Lukasiewicz, T.: Fuzzy description logic programs under the answer set semantics for the semantic web. In: Proceedings of the Second International Conference on Rules and Rule Markup Languages for the Semantic Web (RuleML'06), pp. 89-96 (2006)

53. Lukasiewicz, T., Straccia, U.: Tightly integrated fuzzy description logic programs under the answer set semantics for the semantic web. In: Proceedings of the First International Conference on Web Reasoning and Rule Systems (RR'07), pp. 289-298 (2007)

54. Lukasiewicz, T., Straccia, U.: Top-k retrieval in description logic programs under vagueness for the semantic web. In: Proceedings of the 1st international conference on Scalable Uncertainty Management (SUM'07), pp. 16-30 (2007) 
55. Madrid, N., Ojeda-Aciego, M.: Towards a fuzzy answer set semantics for residuated logic programs. In: Proceedings of the 2008 IEEE/WIC/ACM International Conference on Web Intelligence and Intelligent Agent Technology (WI-IAT'08), pp. 260-264 (2008)

56. Madrid, N., Ojeda-Aciego, M.: On coherence and consistence in fuzzy answer set semantics for residuated logic programs. In: Proceedings of the 8th International Workshop on Fuzzy Logic and Applications (WILF'09), pp. 60-67 (2009)

57. Madrid, N., Ojeda-Aciego, M.: Measuring instability in normal residuated logic programs: discarding information. Communications in Computer and Information Science 80, 128$137(2010)$

58. Madrid, N., Ojeda-Aciego, M.: On the measure of instability in normal residuated logic programs. In: Proceedings of FUZZ-IEEE'10 (2010)

59. Marek, V., Truszczyński, M.: Stable models and an alternative logic programming paradigm, chap. 7, pp. 169-181 (1999)

60. Nerode, A., Remmel, J.B., Subrahmanian, V.S.: Annotated nonmonotonic rule systems. Theoretical Computer Science 171(1-2), 111-146 (1997)

61. Ng, R., Subrahmanian, V.S.: A semantical framework for supporting subjective and conditional probabilities in deductive databases. Journal of Automated Reasoning 10(2), 191-235 (1993)

62. Ng, R., Subrahmanian, V.S.: Stable semantics for probabilistic deductive databases. Information and Computation 110(1), 42-83 (1994)

63. Nicolas, P., Garcia, L., Stéphan, I.: Possibilistic stable models. In: Nonmonotonic Reasoning, Answer Set Programming and Constraints, Dagstuhl Seminar Proceedings (2005)

64. Nicolas, P., Garcia, L., Stéphan, I., Lefèvre, C.: Possibilistic uncertainty handling for answer set programming. Annals of Mathematics and Artificial Intelligence 47(1-2), 139-181 (2006)

65. Niemelä, I.: Logic programs with stable model semantics as a constraint programming paradigm. Annals of Mathematics and Artificial Intelligence 25, 241-273 (1999)

66. Novák, V., Perfilieva, I., Močkor̆, J.: Mathematical Principles of Fuzzy Logic. Kluwer Academic Publishers (1999)

67. Saad, E.: Extended fuzzy logic programs with fuzzy answer set semantics. In: Proceedings of the 3rd International Conference on Scalable Uncertainty Management (SUM'09), pp. 223-239 (2009)

68. Schiex, T., Fargier, H., Verfaillie, G.: Valued constraint satisfaction problems: hard and easy problems. In: Proceedings of the Fourteenth International Joint Conference on Artificial Intelligence (IJCAI'95), pp. 631-637 (1995)

69. Schockaert, S., Janssen, J., Vermeir, D., De Cock, M.: Answer sets in a fuzzy equilibrium logic. In: Proceedings of Rule Reasoning 2009 (RR2009), pp. 135-149 (2009)

70. Shapiro, E.Y.: Logic programs with uncertainties: a tool for implementing rule-based systems. In: Proceedings of the Eighth international joint conference on Artificial intelligence (IJCAI'83), pp. 529-532 (1983)

71. Simons, P.: Extending and implementing the stable model semantics. Ph.D. thesis, Helsinki University of Technology (2000)

72. Straccia, U.: Query answering in normal logic programs under uncertainty. In: In 8th European Conferences on Symbolic and Quantitative Approaches to Reasoning with Uncertainty (ECSQARU-05), pp. 687-700 (2005)

73. Straccia, U.: Annotated answer set programming. In: Proceedings of the 11th International Conference on Information Processing and Management of Uncertainty in KnowledgeBased Systems (IPMU'06) (2006)

74. Straccia, U.: Managing uncertainty and vagueness in description logics, logic programs and description logic programs. In: Reasoning Web: 4th International Summer School 2008, pp. 54-103 (2008)

75. Straccia, U., Ojeda-Aciego, M., Damásio, C.V.: On fixed-points of multivalued functions on complete lattices and their application to generalized logic programs. SIAM Journal on Computing 38(5), 1881-1911 (2009)

76. Subrahmanian, V.S.: Amalgamating knowledge bases. ACM Transactions on Database Systems 19(2), 291-331 (1994)

77. Sugeno, M.: Theory of fuzzy integrals and its application. Ph.D. thesis, Tokyo Institute of Technology (1974)

78. Tarski, A.: A lattice theoretical fixpoint theorem and its application. Pacific Journal of Mathematics 5, 285-309 (1955) 
79. Turksen, I.B.: Interval-valued fuzzy sets and "compensatory and". Fuzzy Sets \& Systems 51(3), 295-307 (1992)

80. Van Nieuwenborgh, D., De Cock, M., Vermeir, D.: An introduction to fuzzy answer set programming. Annals of Mathematics and Artificial Intelligence 50(3-4), 363-388 (2007)

81. Vojtás, P.: Fuzzy logic programming. Fuzzy Sets and Systems 124(3), 361-370 (2001)

82. Wagner, G.: Negation in fuzzy and possibilistic logic programs. Uncertainty Theory in Artificial Intelligence Series (3), 113-128 (1998)

83. Yager, R.R.: A new methodology for ordinal multiple aspect decisions based on fuzzy sets. Decision Sciences 12, 589-600 (1981)

84. Yager, R.R.: On ordered weighted averaging aggregation operators in multicriteria decisionmaking. IEEE Transactions on Systems, Man and Cybernetics 18(1), 183-190 (1988)

85. Yager, R.R., Kacprzyk, J. (eds.): The ordered weighted averaging operators: theory and applications. Kluwer Academic Publishers (1997) 
$\operatorname{about}(P, T) \mid t_{1} t_{2} \quad t_{3} \quad t_{4} t_{5} t_{6} t_{7}$ \begin{tabular}{l|lllllll}
$p_{1}$ & 0.8 & 1 & 0.2 & 0.4 & 0.2 & 0 & 0
\end{tabular} \begin{tabular}{l|lllllllll}
$p_{2}$ & 0 & 0.2 & 0.7 & 0.5 & 0.9 & 0.4 & 0.3
\end{tabular}

$\begin{array}{llllllllll}p_{3} & 1 & 0.1 & 0.1 & 0.2 & 0.3 & 0.2 & 0\end{array}$

$p_{4} \mid \begin{array}{lllllllll}0.5 & 0 & 0 & 1 & 0.2 & 0.4 & 0.8\end{array}$

\begin{tabular}{l|llllllllll}
$p_{5}$ & 0.3 & 0.9 & 0.8 & 0.9 & 0.4 & 0.2 & 0.1
\end{tabular}

\begin{tabular}{l|llllllll}
$p_{6}$ & 0.6 & 0.8 & 0.3 & 0.7 & 0.8 & 0.3 & 0
\end{tabular}

$\begin{array}{llllllll}p_{7} & 0 & 1 & 1 & 1 & 0 & 0.3 & 0.1\end{array}$

$\begin{array}{lllllllll}p_{8} & 0.9 & 0.2 & 0.1 & 0.7 & 0 & 0.1 & 1\end{array}$

\begin{tabular}{l|lllllll}
$p_{9}$ & 0.1 & 0.7 & 0.1 & 0.2 & 0.1 & 1 & 0.7
\end{tabular}

\begin{tabular}{l|lllllll}
$p_{10}$ & 0.7 & 0.6 & 0.6 & 0.3 & 0.2 & 1 & 0.1
\end{tabular}

Table 3: about $(P, T)$ scores

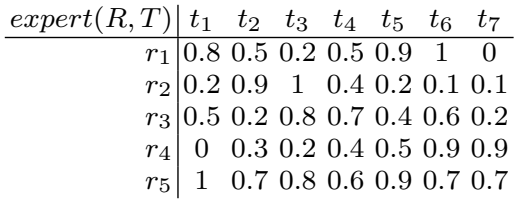

Table 4: $\operatorname{expert}(R, T)$ scores

close $\left(U, U^{\prime}\right) \mid \begin{array}{llllllllll}u_{1} & u_{2} & u_{3} & u_{4} & u_{5} & u_{6} & u_{7} & u_{8} & u_{9} & u_{10}\end{array}$

\begin{tabular}{l|lllllllllll}
$u_{1}^{\prime}$ & 0.5 & 0.3 & 0.4 & 0.8 & 0.1 & 1 & 0.4 & 0.3 & 0.9 & 0.4
\end{tabular}

$\begin{array}{llllllllllll}u_{2}^{\prime} & 0.5 & 0.8 & 0.5 & 0.4 & 0.4 & 0.5 & 0.1 & 0 & 0.4 & 0.1\end{array}$

\begin{tabular}{l|lllllllllll}
$u_{3}^{\prime}$ & 0 & 0 & 0 & 1 & 0.3 & 0.6 & 0.3 & 0.9 & 0.2 & 0.1
\end{tabular}

\begin{tabular}{l|llllllllll}
$u_{4}^{\prime}$ & 1 & 0.9 & 0.4 & 0.2 & 0.1 & 1 & 0.5 & 0.2 & 0.9 & 1
\end{tabular}

\begin{tabular}{l|llllllllll}
$u_{5}^{\prime}$ & 0.8 & 0.1 & 0.1 & 0.4 & 0.2 & 0.3 & 1 & 0.3 & 0.1 & 0.2
\end{tabular}

Table 5: $\operatorname{close}\left(U, U^{\prime}\right)$ scores

$\operatorname{conflict}(R, P) \mid p_{1} \quad p_{2} \quad p_{3} \quad p_{4} \quad p_{5} \quad p_{6} \quad p_{7} \quad p_{8} \quad p_{9} \quad p_{10}$ $\begin{array}{lllllllllllll}r_{1} & 0.5 & 0.3 & 0.4 & 0.8 & 0.1 & 1 & 0.4 & 0.3 & 0.9 & 0.4\end{array}$ $\begin{array}{llllllllllll}r_{2} & 0.5 & 0.8 & 0.5 & 0.4 & 0.4 & 0.5 & 0.1 & 0 & 0.4 & 0.1\end{array}$ $\begin{array}{llllllllllll}r_{3} & 0 & 0 & 0 & 1 & 0.3 & 0.6 & 0.3 & 0.9 & 0.2 & 0.1\end{array}$ $\begin{array}{lllllllllll}r_{4} & 1 & 0.9 & 0.4 & 0.2 & 0.1 & 1 & 0.5 & 0.2 & 0.9 & 1\end{array}$

$\begin{array}{llllllllllll}r_{5} & 0.8 & 0.1 & 0.1 & 0.4 & 0.2 & 0.3 & 1 & 0.3 & 0.1 & 0.2\end{array}$

Table 6: $\operatorname{conflict}(R, P)$ scores

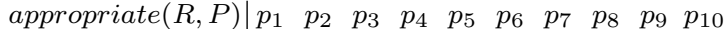
\begin{tabular}{l|lllllllllll}
$r_{1}$ & 0.5 & 0.7 & 0.6 & 0.2 & 0.4 & 0 & 0.5 & 0.7 & 0.1 & 0.6
\end{tabular} $\begin{array}{lllllllllllll}r_{2} & 0.5 & 0.2 & 0.2 & 0.4 & 0.6 & 0.5 & 0.9 & 0.1 & 0.6 & 0.6\end{array}$ \begin{tabular}{l|lllllllllll}
$r_{3}$ & 0.3 & 0.5 & 0.5 & 0 & 0.6 & 0.4 & 0.7 & 0.1 & 0.6 & 0.6
\end{tabular} \begin{tabular}{l|llllllllll}
$r_{4}$ & 0 & 0.1 & 0.1 & 0.7 & 0.3 & 0 & 0.4 & 0.8 & 0.1 & 0
\end{tabular} \begin{tabular}{l|llllllllll}
$r_{5}$ & 0.2 & 0.8 & 0.9 & 0.6 & 0.6 & 0.7 & 0 & 0.7 & 0.7 & 0.7
\end{tabular}

Table 7: appropriate $(R, P)$ scores 


\begin{tabular}{r|c|c} 
assignments & $A_{1}$ & $A_{2}$ \\
\hline$p_{1}$ & $r_{1}, r_{2}, r_{3}$ & $r_{1}, r_{2}, r_{4}$ \\
$p_{2}$ & $r_{1}, r_{3}, r_{5}$ & $r_{1}, r_{3}, r_{4}$ \\
$p_{3}$ & $r_{1}, r_{3}, r_{5}$ & $r_{1}, r_{4}, r_{5}$ \\
$p_{4}$ & $r_{2}, r_{4}, r_{5}$ & $r_{2}, r_{4}, r_{5}$ \\
$p_{5}$ & $r_{2}, r_{3}, r_{5}$ & $r_{2}, r_{3}, r_{5}$ \\
$p_{6}$ & $r_{2}, r_{3}, r_{5}$ & $r_{2}, r_{3}, r_{5}$ \\
$p_{7}$ & $r_{1}, r_{2}, r_{3}$ & $r_{1}, r_{2}, r_{3}$ \\
$p_{8}$ & $r_{1}, r_{4}, r_{5}$ & $r_{1}, r_{4}, r_{5}$ \\
$p_{9}$ & $r_{2}, r_{3}, r_{5}$ & $r_{2}, r_{3}, r_{5}$ \\
$p_{10}$ & $r_{1}, r_{3}, r_{5}$ & $r_{1}, r_{3}, r_{5}$
\end{tabular}

Table 8: Assignments for answer sets $A_{1}$ and $A_{2}$

\begin{tabular}{r|cccccccccc} 
inappropriate $(R, P)$ & $p_{1}$ & $p_{2}$ & $p_{3}$ & $p_{4}$ & $p_{5}$ & $p_{6}$ & $p_{7}$ & $p_{8}$ & $p_{9}$ & $p_{10}$ \\
\hline$r_{1}$ & 0 & 0 & 0 & 0.8 & 0.6 & 1 & 0 & 0 & 0.9 & 0 \\
$r_{2}$ & 0 & 0.8 & 0.8 & 0 & 0 & 0 & 0 & 0.9 & 0 & 0.4 \\
$r_{3}$ & 0 & 0 & 0 & 1 & 0 & 0 & 0 & 0.9 & 0 & 0 \\
$r_{4}$ & 1 & 0.9 & 0.9 & 0 & 0.7 & 1 & 0.6 & 0 & 0.9 & 1 \\
$r_{5}$ & 0.8 & 0 & 0 & 0 & 0 & 0 & 1 & 0 & 0 & 0
\end{tabular}

Table 9: inappropriate $(R, P)$ scores for $A_{1}$

\begin{tabular}{r|cccccccccc} 
inappropriate $(R, P)$ & $p_{1}$ & $p_{2}$ & $p_{3}$ & $p_{4}$ & $p_{5}$ & $p_{6}$ & $p_{7}$ & $p_{8}$ & $p_{9}$ & $p_{10}$ \\
\hline$r_{1}$ & 0 & 0 & 0 & 0.8 & 0.6 & 1 & 0 & 0 & 0.9 & 0 \\
$r_{2}$ & 0 & 0.8 & 0.8 & 0 & 0 & 0 & 0 & 0.9 & 0 & 0.4 \\
$r_{3}$ & 0.7 & 0 & 0.5 & 1 & 0 & 0 & 0 & 0.9 & 0 & 0 \\
$r_{4}$ & 0 & 0 & 0 & 0 & 0.7 & 1 & 0.6 & 0 & 0.9 & 1 \\
$r_{5}$ & 0.8 & 0.2 & 0 & 0 & 0 & 0 & 1 & 0 & 0 & 0
\end{tabular}

Table 10: inappropriate $(R, P)$ scores for $A_{2}$ 